\begin{tabular}{|c|l|}
\hline Title & A bsolutely continuous invariant measures for expanding piecewise linear maps \\
\hline Author(s) & Tsujii, M. \\
\hline Citation & Hokkaido University Preprint Series in Mathematics, 442, 1-28 \\
\hline Issue Date & 1999-1-1 \\
\hline DOI & 10.14943/83588 \\
\hline Doc URL & http://hdl.handle.net/2115/69192 \\
\hline Type & bulletin (article) \\
\hline File Information & pre442.pdf \\
\hline
\end{tabular}

Instructions for use 


\title{
Absolutely continuous invariant measures for expanding piecewise linear maps
}

\author{
Masato Tsujii
}

Series \#442. January 1999 


\section{HOKKAIDO UNIVERSITY PREPRINT SERIES IN MATHEMATICS}

\#417 K. Yamaguchi, $G_{2}$-Geometry of overdetermined systems of second order, 22 pages. 1998.

\#418 M. Ishikawa and S. Matsui, Existence of a forward self-similar stagnation flow of the Navier-Stokes equations, 8 pages. 1998.

\#419 S. Izumiya, H. Katsumi and T. Yamasaki, The rectifying developable and the spherical Darboux image of a space curve, 16 pages. 1998.

\#420 R. Kobayashi and Y. Giga, Equations with singular diffusivity, 45 pages. 1998.

\#421 D. Pei and T. Sano, The focal developable and the binormal indicatrix of a nonlightlike curve in Minkowski 3-space, 14 pages. 1998.

\#422 R. Kobayashi, J. A. Warren and W. C. Carter, Modeling grain boundaries using a phase field technique, 12 pages. 1998.

\#423 T. Tsukada, Reticular Legendrian Singularities, 28 pages. 1998.

\#424 A. N. Kirillov and M. Shimozono, A generalization of the Kostka-Foulkes polynomials, 37 pages. 1998.

\#425 M. Nakamura, Strichartz estimates for wave equations in the homogeneous Besov space, 17 pages. 1998.

\#426 A. Arai, On the essential spectra of quantum field Hamiltonians, 18 pages. 1998.

\#427 T. Sano, Bifurcations of affine invariants for one parameter family of generic convex plane curves, 11 pages. 1998.

\#428 F. Hiroshima, Ground states of a model in quantum electrodynamics, 48 pages. 1998.

\#429 F. Hiroshima, Uniqueness of the ground state of a model in quantum electrodynamics: A functional integral approach, 32 pages. 1998.

\#430 J. F. Van Diejen and A. N. Kirillov, Formulas for $q$-spherical functions using inverse scattering theory of reflectionless Jacobi operators, 33 pages. 1998.

\#431 G. Ishikawa, Determinacy, transversality and Lagrange stability, 13 pages. 1998.

\#432 T. Yoshida, Categorical aspects of generating functions(II): Operations on categories and functors, 65 pages. 1998.

\#433 K. Ito, Loss of convexity of compact hypersurfaces moved by surface diffusion, 20 pages. 1998.

\#434 Y. Shimizu, $L^{\infty}$-estimate of first-order space derivatives of Stokes flow in a half space, 22 pages. 1998.

\#435 T. Uemura, Morita-Mumford classes on finite cyclic subgroups of the mapping class group of closed surfaces, 14 pages. 1998.

\#436 A. Inoue and H. Kikuchi, Abel-Tauber theorems for Hankel and Fourier transforms and a problem of Boas, 20 pages. 1998.

\#437 T. Nakazi and T. Osawa, Finite rank intermediate Hankel operators on the Bergman space, 16 pages. 1998.

\#438 R. Yoneda, Compact Toeplitz operators on Bergman spaces, 14 pages. 1998.

\#439 A. Inoue and Y. Kasabara, On the asymptotic behavior of the prediction error of a stationary process, 12 pages. 1998.

\#440 A. Arai, M. Hirokawa and F. Hiroshima, On the absence of eigenvectors of Hamiltonians in a class of massless quantum field models without infrared cutoff, 26 pages. 1998.

\#441 A. N. Kirillov, A. Schilling and M. Shimozono, A bijection between Littlewood-Richardson tableaux and rigged configurations, 66 pages. 1999. 


\title{
Absolutely continuous invariant measures for expanding piecewise linear maps
}

\author{
Masato TSUJII* \\ Dedicated to \\ Professor Takuo Fukuda \\ on his 60 th birthday
}

\begin{abstract}
We prove the existence of absolutely continuous invariant measures for expanding piecewise linear maps on bounded polyhedral domains of arbitrary dimensions.
\end{abstract}

\section{Introduction}

In this paper, we study ergodic properties of expanding piecewise linear maps defined on bounded polyhedral domains in Euclidean space $\mathbb{R}^{d}$. Especially, we prove the existence of absolutely continuous invariant measures for arbitrary expanding piecewise linear maps. Formerly, it was known that almost all expanding piecewise linear maps admit absolutely continuous invariant measures[3]. In the case of dimension $d=2$, it was also known that all expanding piecewise linear maps (or all expanding piecewise real analytic maps, more generally) admit absolutely continuous invariant measures $[2,11]$. We extend these results and complete the argument on the existence of absolutely continuous invariant measures for expanding piecewise linear maps. See $[1,2,3,4,7,8,9,10,11,12]$ for more information about ergodic properties of expanding piecewise smooth maps.

Each connected component of complements of hyperplanes in $\mathbb{R}^{d}$ is called a half-space. A closed subset $A \subset \mathbb{R}^{d}$ is said to be a polyhedron if it is obtained from closures of halfspaces by taking intersection and union for finite times. Notice that we do not assume polyhedra to be bounded nor connected in this paper.

*Department of Mathematics, Hokkaido University, Sapporo, 060-0810, JAPAN, e-mail: tsujii@math.sci.hokudai.ac.jp 
Definition Let $U$ be a bounded polyhedron in $\mathbb{R}^{d}$ with non-empty interior. An expanding piecewise linear map on $U$ is a combination $(\mathcal{T}, \mathcal{U})$ of a map $\mathcal{T}: U \rightarrow U$ and a family $\mathcal{U}=\left\{U_{k}\right\}_{k=1}^{\ell}$ of polyhedra $U_{k} \subset U, k=1,2, \ldots, \ell$, satisfying the conditions

(i) the interiors of polyhedra $U_{k}$ are mutually disjoint,

(ii) $\cup_{k=1}^{\ell} U_{k}=U$, and

(iii) the restriction of the map $\mathcal{T}$ to the interior of each $U_{k}$ is an affine map.

The map $\mathcal{T}$ itself in the above definition will be called a piecewise linear map if the associated partition is clear. Let us denote $U^{\circ}=\cup_{k=1}^{\ell} \operatorname{in} t U_{k}$. A piecewise linear map $(\mathcal{T}, \mathcal{U})$ is expanding if there exists a constant $\rho>1$ such that

$$
\left\|D \mathcal{T}_{x}(v)\right\| \geq \rho\|v\| \quad \text { for all } x \in U^{\circ} \text { and all } v \in T_{x} \mathbb{R}^{d} .
$$

We denote, by $\rho(\mathcal{T})$, the maximum of numbers $\rho$ satisfying (1).

The main result of this paper is

Theorem 1 An arbitrary expanding piecewise linear map adimits an absolutely continuous invariant probability measure.

We prove theorem 1 by showing a version of Lasota-Yorke type inequality for the so-called generalized variations[8]. It is known that many other ergodic properties of follows from that kind of inequality. (See [8].) For example, we have

Theorem 2 Let $(\mathcal{T}, \mathcal{U})$ be an expanding piecewise linear maps on $U \subset \mathbb{R}^{d}$. Then

(i) there exist finitely many absolutely continuous ergodic probability measures $\mu_{i}, 1 \leq$ $i \leq p$, for $\mathcal{T}$, and all absolutely continuous invariant probability measures for $\mathcal{T}$ are convex combinations of them, and

(ii) the basin of each measure $\mu_{i}$, that is,

$$
\operatorname{Basin}\left(\mu_{i}\right)=\left\{\begin{array}{l|l}
x \in U & \frac{1}{n} \sum_{j=1}^{n-1} \delta_{\mathcal{T}^{j}(x)} \rightarrow \mu_{i} \text { weakly. }
\end{array}\right\}
$$

is an open set modulo sets with Lebesgue measure 0 , and the union $\cup_{i=1}^{p} \operatorname{Basin}\left(\mu_{i}\right)$ has full Lebesgue measure in $U$.

Though theorem 2 is essentially contained in [7], [10] and [3], we will give a proof of theorem2 in the last section. For more detailed properties such as exponential decay of correlation, weak Bernoulli property and central limit theorems, we refer the argument in $[5,7,10]$. 
In this paper, we proceed as follows. In section 2 , we define a quantity $\mathcal{M}(\mathcal{T})$ for each piecewise linear map $\mathcal{T}$, which measures the local complexity of the partition $\mathcal{P}$ from the view point of dynamics. We call $\mathcal{M}(\mathcal{T})$ weighted multiplicity. In section 3 , we will prove the existence of an absolutely continuous invariant measure for $\mathcal{T}$ under an additional condition

$$
C(d) \mathcal{M}(\mathcal{T})+\rho(\mathcal{T})^{-1}<1
$$

where $C(d)$ is a constant that depends only on the dimension $d$. This part heavily depends on a recent work of B. Saussol[10]. In section 4, we will give a proof of the theorem of Saussol that we used in section 3 .

A piecewise linear map admits an absolutely continuous invariant measure if and only if one of its iterations does. So our main task is confirming that one of iterations of an arbitrary expanding piecewise linear map $\mathcal{T}$ satisfies the condition (2). This is the part where this paper mainly concerns. Since $\rho\left(\mathcal{T}^{n}\right)^{-1} \rightarrow 0$ as $n \rightarrow \infty$ obviously, it is enough to check

$$
\liminf _{n \rightarrow \infty} \mathcal{M}\left(\mathcal{T}^{n}\right)=0
$$

This will be proved in section 8 after preparations in sections 5,6 and 7 . In section 9 , we prove theorem 2 .

Remark (1) In the argument below, we assume that each $U_{k}$ in the partition is convex and has non-empty interior. Since we can make any piecewise linear maps $(\mathcal{T}, \mathcal{U})$ satisfy this assumption by discarding some of $U_{k}$ 's and by cutting $U_{k}$ 's by hyperplanes, this assumption does not lessen generality of our argument. (2) The values of an expanding piecewise linear map $\mathcal{T}$ on the boundaries of $U_{k}$ 's has nothing to do with the results of theorem 1 and 2 above. Hence, we will consider the map $\mathcal{T}$ to be defined only on $U^{\circ}=\cup_{k} \operatorname{int}\left(U_{k}\right)$ and not defined on the complement $U \backslash U^{\circ}$, for simplicity.

\section{Weighted multiplicity}

Let $d(\cdot, \cdot)$ be Euclidean distance on $\mathbb{R}^{d}$ and let $\boldsymbol{H}^{m}$ be $m$-dimensional Hausdorff measure on $\mathbb{R}^{d}$. We denote the open ball with the radius $\eta>0$ and the center $x \in \mathbb{R}^{d}$ by

$$
B(x, \eta)=\left\{y \in \mathbb{R}^{d} \mid d(x, y)<\eta\right\} .
$$

A subset $A \subset \mathbb{R}^{d}$ is said to be a cone in a ball $B(x, \eta)$ if any segments

$$
L_{y}:=\{x+t(y-x) \mid t \geq 0\} \cap B(x, a)
$$

for $y \in A \cap B(x, a)$ are contained in $A$. We say that a ball $B(x, a)$ has regular cone property if all $U_{k}, 1 \leq k \leq \ell$, are cones in it. Notice that, for each $x \in U$, we can choose small number $\eta>0$ so that $B(x, \eta)$ has regular cone property. 
For vectors $a \neq 0$ and $b \neq 0$ in $\mathbb{R}^{d}$, let $\angle(a, b)$ be the angle between them. For $x \in U$, $v \in \mathbb{R}^{d} \backslash\{0\}, 0 \leq \theta \leq \pi$ and $\delta>0$, we put

$$
\text { Cone }(x, \delta ; v, \theta)=\left\{y \in \mathbb{R}^{d} \mid 0<\|y-x\| \leq \delta \text { and } \angle(v, y-x) \leq \theta\right\}
$$

and

$$
\mathrm{S}(x, \delta ; v, \theta)=\left\{y \in \mathbb{R}^{d} \mid\|y-x\|=\delta \text { and } \angle(v, y-x) \leq \theta\right\} .
$$

Let $T_{k}: \mathbb{R}^{d} \rightarrow \mathbb{R}^{d}$ be the affine map that coincides with $\mathcal{T}$ on the interior of $U_{k}$. For a subset $B \subset \mathbb{R}^{d}$, we denote its boundary in $\mathbb{R}^{d}$ by $\partial B$. Then, for $1 \leq k \leq \ell$ and $\varepsilon>0$, we put

$$
E(k, \varepsilon)=\left\{y \in \mathbb{R}^{d} \mid d\left(T_{k}(y), T_{k}(z)\right)<\varepsilon \text { for some point } z \in \partial U_{k} .\right\} .
$$

For $x \in U, \varepsilon>0, v \in \mathbb{R}^{d} \backslash\{0\}$ and $0 \leq \theta<\pi$, let us consider the sum of ratios

$$
\sum_{k=1}^{\ell} \frac{\boldsymbol{H}^{d}(E(k, \varepsilon \delta \theta) \cap \operatorname{Cone}(x, \delta ; v, \theta))}{\varepsilon \cdot \boldsymbol{H}^{d}(\operatorname{Cone}(x, \delta ; v, \theta))} .
$$

This quantity depends on a positive number $\delta>0$. But we have

Lemma 3 The quantity (3) takes a constant value for sufficiently small $\delta>0$.

Proof. Take $\delta_{0}>0$ so small that $B\left(x,(1+4 \pi) \delta_{0}\right)$ has regular cone property. Consider a map

$$
\xi_{x, t}: \mathbb{R}^{d} \rightarrow \mathbb{R}^{d}, \quad \xi_{t}(y)=x+t(y-x)
$$

Then we have

$$
\xi_{x, \delta / \delta_{0}}\left(E\left(k, \varepsilon \delta_{0} \theta\right) \cap \operatorname{Cone}\left(x, \delta_{0} ; v, \theta\right)\right)=E(k, \varepsilon \delta \theta) \cap \operatorname{Cone}(x, \delta ; v, \theta)
$$

for $0<\delta \leq \delta_{0}$. In fact, if we take a point $y \in E\left(k, \varepsilon \delta_{0} \theta\right) \cap \operatorname{Cone}\left(x, \delta_{0} ; v, \theta\right)$, we can find a point $z \in \partial U_{k}$ such that $d\left(T_{k}(y), T_{k}(z)\right)<\varepsilon \delta_{0} \theta$. Then $d(y, z) \leq \rho(\mathcal{T})^{-1} \varepsilon \delta_{0} \theta<4 \pi \delta_{0}$ and $z \in B\left(x,(1+4 \pi) \delta_{0}\right)$. Obviously we have $d\left(T_{k}\left(\xi_{x, \delta / \delta_{0}}(y)\right), T_{k}\left(\xi_{x, \delta / \delta_{0}}(z)\right)\right)<\varepsilon \delta \theta$ and $\xi_{x, \delta / \delta_{0}}(z)$ belongs to $\partial U_{k}$ from regular cone property of the ball $B\left(x,(1+4 \pi) \delta_{0}\right)$. This means that $\xi_{x, \delta / \delta_{0}}(y)$ is contained in $E(k, \varepsilon \delta \theta)$. Hence the left hand side of the equality $(5)$ is contained in the right hand side. The reverse inclusion can be shown in similar way. Since $\xi_{x, \delta / \delta_{0}}$ is an affine map and maps $\operatorname{Cone}\left(x, \delta_{0} ; v, \theta\right)$ onto Cone $(x, \delta ; v, \theta)$, the equality (5) implies that (3) takes the same value for $0<\delta \leq \delta_{0}$ and $\delta_{0}$.

We denote this constant value by $\mu(\mathcal{T}, x, \varepsilon ; v, \theta)$. In other words, we set

$$
\mu(\mathcal{T}, x, \varepsilon ; v, \theta)=\lim _{\delta \rightarrow+0} \sum_{k=1}^{\ell} \frac{\boldsymbol{H}^{d}(E(k, \varepsilon \delta \theta) \cap \operatorname{Cone}(x, \delta ; v, \theta))}{\varepsilon \cdot \boldsymbol{H}^{d}(\operatorname{Cone}(x, \delta ; v, \theta))} .
$$


If $x \in U^{\circ}, \mu(\mathcal{T}, x, \varepsilon ; v, \theta)=0$ obviously.

Weighted multiplicity of a piecewise linear map $\mathcal{T}$ at a point $x \in U$ is defined as

$$
\mathcal{M}(\mathcal{T}, x)=\sup _{0<\varepsilon \leq 4} \sup _{v \in \mathbb{R}^{d} \backslash\{0\}} \sup _{0<\theta<\pi} \mu(\mathcal{T}, x, \varepsilon ; v, \theta) .
$$

Remark that the supremum with respect to $\varepsilon$ is taken over $0<\varepsilon \leq 4$. The bound 4 comes from very technical reason and does not have any special meaning. Finally, we put

$$
\mathcal{M}(\mathcal{T})=\sup _{x \in U} \mathcal{M}(\mathcal{T}, x)
$$

and call it weighted multiplicity of a piecewise linear map $\mathcal{T}$.

\section{An existence theorem for absolutely continuous invariant probability measures}

In this section we prove

Theorem 4 There exists a constant $C(d)>0$, which depends only on the dimension $d$, such that, if $\rho(\mathcal{T})>2$ and

$$
C(d) \mathcal{M}(\mathcal{T})+\rho(\mathcal{T})^{-1}<1
$$

there exists an absolutely continuous invariant probability measure for $\mathcal{T}$.

We derive this theorem from a recent result of $B$. Saussol in [10]. For $\varepsilon>0$ and $\eta>0$, we define

$$
G(\eta, \varepsilon, x)=\sum_{k=1}^{\ell} \frac{\boldsymbol{H}^{d}(E(k, \varepsilon) \cap B(x, \eta))}{\boldsymbol{H}^{d}(B(x, \eta))}
$$

and

$$
G(\eta, \varepsilon)=\sup _{x \in \mathbb{R}^{d}} G(\eta, \varepsilon, x) .
$$

Remark that the supremum is taken over $x \in \mathbb{R}^{d}$ and not only over $x \in U$. For the simplicity of the notation, we denote $s=\rho(\mathcal{T})^{-1}$. The following theorem is a special case of a theorem due to B.Saussol[10, Proposition 6.2].

Theorem 5 If we have

$$
\Gamma:=s+\sup _{0<\varepsilon \leq \eta}\left(\frac{\eta}{\varepsilon} \cdot G((1-s) \eta, \varepsilon)\right)<1
$$

for some $\eta>0$, there exists an absolutely continuous invariant probability measure for $\mathcal{T}$. 
We will give a proof of this theorem in section 4 for completeness. From theorem 5, we have only to prove

Proposition 6 There exists a constant $C(d)$, which depend only on the dimension $d$, such that we have

$$
\sup _{0<\varepsilon \leq \eta}\left(\frac{\eta}{\varepsilon} \cdot G((1-s) \eta, \varepsilon)\right) \leq C(d) \mathcal{M}(\mathcal{T})
$$

if $\rho(\mathcal{T})>2$ and if $\eta>0$ is sufficiently small.

For the proof of proposition 6 , we prepare one elementary lemma.

Lemma 7 If we take $r>0$ sufficiently small, we can choose a point $p(x) \in \mathbb{R}^{d}$ for each $x \in \mathbb{R}^{d}$ such that $B(p(x),\|x-p(x)\|+r)$ has regular cone property.

Proof. For $0 \leq j \leq d-1$, let $F_{j}$ be the union of $j$-dimensional faces of the polyhedra $U_{k}$, $1 \leq k \leq \ell$. For $x \in U$, let us denote, by $\eta(x)$, the maximum of number $\eta$ such that $B(x, \eta)$ has regular cone property. Then it is not difficult to see that $\eta(x)$ is a positive-valued lower semicontinuous function over $F_{j} \backslash F_{j-1}, 1 \leq j \leq d-1$.

First, take $r_{0}>0$ so small that the balls $B\left(x, 2 r_{0}\right)$ for $x \in F_{0}$ satisfy regular cone property. Then put $D_{0}=\bigcup_{x \in F_{0}} B\left(x, r_{0}\right)$. Inductively, we define $r_{j}>0$ and open sets $D_{j}$ for $1 \leq j \leq d-1$ so that $\cup_{i \leq j} D_{i}$ is an open neighborhood of $F_{j}$. Assume that we have chosen $r_{i}$ and $D_{i}$ for $0 \leq i \leq j-1$. Then since the function $\eta(\cdot)$ is positive-valued and lower semicontinuous on $F_{j} \backslash \bigcup_{i=0}^{j-1} D_{i}$, we can choose a number $0<r_{j}<r_{j-1}$ such that $B\left(x, 2 r_{j}\right)$ for $x \in F_{j} \backslash \bigcup_{i=0}^{j-1} D_{i}$ has regular cone property. Put

$$
D_{j}=\left\{y \in \mathbb{R}^{d} \mid d(y, z)<r_{j} \text { for some } z \in F_{j} \backslash \cup_{i=0}^{j-1} D_{i} \cdot\right\} .
$$

Obviously, $\cup_{i=0}^{j} D_{i}$ is an open neighborhood of $F_{j}$.

From the above definition, $\cup_{j=0}^{d-1} D_{j}$ contains a neighborhood of $F_{d-1}=\cup_{k=1}^{\ell} \partial U_{k}$. Choose $0<r<r_{d-1}$ so small that the $r$-neighborhood of $F_{d-1}$ is contained in $\cup_{j=0}^{d-1} D_{j}$. If $x \in D_{j}$ for some $0 \leq j<d$, there exists a point $p(x) \in F_{j} \backslash \bigcup_{i=0}^{j-1} D_{i}$ such that $x \in B\left(p(x), r_{j}\right)$. From the choice of $r_{j}$, the ball

$$
B(p(x),\|x-p(x)\|+r) \subset B\left(p(x), 2 r_{j}\right)
$$

has regular cone property. Otherwise, put $p(x)=x$, then $B(p(x),\|x-p(x)\|+r)=$ $B(p(x), r)$ does not intersect $F_{d-1}$ and has regular cone property in trivial sense.

Proof of proposition 6 Let $r>0$ and $p(\cdot)$ be those in the last lemma. We show that the conclusion of proposition 6 holds for $0<\eta<r / 3$. Let us consider a point $x \in \mathbb{R}^{d}$ and numbers $0<\varepsilon \leq \eta$. Put $\eta^{\prime}=(1-s) \eta$ for simplicity of the notation. Notice that $\eta^{\prime}>\eta / 2$ from the assumption $\rho(\mathcal{T})>2$. For the map $\xi_{p(x), t}(y)=t(y-p(x))+p(x)$, we can show 
Lemma 8 If a point $w \in \mathbb{R}^{d}$ is contained in $E(k, \varepsilon) \cap B\left(p(x),\|x-p(x)\|+2 \eta^{\prime}\right)$, the image $\xi_{p(x), t}(w)$ belongs to $E(k, t \varepsilon)$ for $0 \leq t \leq 1$.

Proof. For any point $w \in E(k, \varepsilon) \cap B\left(p(x),\|x-p(x)\|+2 \eta^{\prime}\right)$, there exists a point $z \in \partial U_{k}$ such that $d\left(T_{k}(w), T_{k}(z)\right) \leq \varepsilon$. Then we have $d(w, z) \leq \rho(\mathcal{T})^{-1} \varepsilon<\varepsilon / 2 \leq \eta / 2 \leq \eta^{\prime}$ and

$$
d(z, p(x)) \leq d(z, w)+d(w, p(x))<\|x-p(x)\|+3 \eta^{\prime}<\|x-p(x)\|+r .
$$

From regular cone property of the ball $B(p(x),\|x-p(x)\|+r)$, the point $\xi_{t}(z)$ is contained in $\partial U_{k}$. Since $d\left(T_{k}\left(\xi_{t}(w)\right), T_{k}\left(\xi_{t}(z)\right)\right) \leq t \varepsilon$, the point $\xi_{t}(w)$ belongs to $E(k, t \varepsilon)$.

Now let us consider two cases: $\eta^{\prime} \geq\|x-p(x)\| / 2$ or otherwise. First we consider the case where $\eta^{\prime} \geq\|x-p(x)\| / 2$. Let us put $\tilde{\eta}=\|x-p(x)\|+\eta^{\prime} \leq 3 \eta^{\prime}$. Since the ball $B\left(x, \eta^{\prime}\right)$ is contained in $B(p(x), \tilde{\eta})$, we have

$$
G\left(\eta^{\prime}, \varepsilon, x\right) \leq \sum_{k=1}^{\ell} \frac{\boldsymbol{H}^{d}(E(k, \varepsilon) \cap B(p(x), \tilde{\eta}))}{\boldsymbol{H}^{d}\left(B\left(x, \eta^{\prime}\right)\right)} .
$$

From lemma 8, it holds

$$
\begin{aligned}
\sum_{k=1}^{\ell} \frac{\boldsymbol{H}^{d}(E(k, \varepsilon) \cap B(p(x), \tilde{\eta}))}{\boldsymbol{H}^{d}(B(x, \tilde{\eta}))} & \leq \lim _{t \rightarrow 0} \sum_{k=1}^{\ell} \frac{\boldsymbol{H}^{d}(E(k, t \varepsilon) \cap B(p(x), t \tilde{\eta}))}{\boldsymbol{H}^{d}(B(x, t \tilde{\eta}))} \\
& =\left(\frac{\varepsilon}{\pi \tilde{\eta}}\right) \cdot \mu\left(\mathcal{T}, p(x), \frac{\varepsilon}{\pi \tilde{\eta}} ; v, \pi\right) .
\end{aligned}
$$

Since $(1 / 2) \eta \leq \eta^{\prime} \leq \tilde{\eta} \leq 3 \eta^{\prime}$, this implies

$$
\frac{\eta}{\varepsilon} \cdot G\left(\eta^{\prime}, \varepsilon, x\right) \leq \frac{\eta}{\varepsilon} \cdot \frac{\varepsilon}{\pi \tilde{\eta}} \cdot \frac{\boldsymbol{H}^{d}(B(x, t \tilde{\eta}))}{\boldsymbol{H}^{d}\left(B\left(x, t \eta^{\prime}\right)\right)} \cdot \mu\left(\mathcal{T}, p(x), \frac{\varepsilon}{\pi \tilde{\eta}} ; v, \pi\right)<3^{d} \mathcal{M}(\mathcal{T}) .
$$

Next, let us consider the case where $\eta^{\prime}<\|x-p(x)\| / 2$. We put

$$
\theta=\arcsin \left(\eta^{\prime} /\|x-p(x)\|\right)<\pi / 6, \quad v=x-p(x), \quad \text { and } \quad R=\|x-p(x)\|-\eta^{\prime} .
$$

Then $2 R \geq\|x-p(x)\|$ and $2 R \theta \geq\|x-p(x)\| \theta \geq \eta^{\prime}$. Since $B\left(x, \eta^{\prime}\right)$ is contained in

$$
\text { Cone }\left(p(x), R+2 \eta^{\prime} ; v, \theta\right) \cap\left\{y \in \mathbb{R}^{d} \mid R \leq\|y-p(x)\| \leq R+2 \eta^{\prime}\right\},
$$

we have,

$$
\boldsymbol{H}^{d}\left(E(k, \varepsilon) \cap B\left(x, \eta^{\prime}\right)\right) \leq \int_{R}^{R+2 \eta^{\prime}} \boldsymbol{H}^{d-1}(E(k, \varepsilon) \cap \mathrm{S}(p(x), a ; v, \theta)) d a
$$


From lemma 8, we have, for $R \leq a \leq R+2 \eta^{\prime}$ and $0 \leq t \leq 1$,

$$
\begin{aligned}
t^{d-1} \boldsymbol{H}^{d-1}(E(k, \varepsilon) \cap \mathrm{S}(p(x), a ; v, \theta)) & \leq \boldsymbol{H}^{d-1}(E(k, t \varepsilon) \cap \mathrm{S}(p(x), t a ; v, \theta)) \\
& \leq \boldsymbol{H}^{d-1}(E(k, \varepsilon) \cap \mathrm{S}(p(x), t a ; v, \theta))
\end{aligned}
$$

Applying (11) to (10) with $t=R / a$, we obtain

$$
\begin{aligned}
\boldsymbol{H}^{d}\left(E(k, \varepsilon) \cap B\left(x, \eta^{\prime}\right)\right) & \leq \int_{R}^{R+2 \eta^{\prime}} \boldsymbol{H}^{d-1}(E(k, \varepsilon) \cap \mathrm{S}(p(x), R ; v, \theta))\left(\frac{a}{R}\right)^{d-1} d a \\
& \leq 2 \eta^{\prime} \cdot 3^{d-1} \cdot \boldsymbol{H}^{d-1}(E(k, \varepsilon) \cap \mathrm{S}(p(x), R ; v, \theta)) .
\end{aligned}
$$

Again applying (11) with $a=R$ and $t=b / R$, we have, for $0<t \leq 1$,

$$
\begin{aligned}
\boldsymbol{H}^{d}(E(k, t \varepsilon) \cap \operatorname{Cone}(p(x), t R ; v, \theta)) \\
\quad=\int_{0}^{t R} \boldsymbol{H}^{d-1}(E(k, t \varepsilon) \cap \mathrm{S}(p(x), b ; v, \theta)) d b \\
\geq \int_{0}^{t R}(b / R)^{d-1} \boldsymbol{H}^{d-1}(E(k, \varepsilon) \cap \mathrm{S}(p(x), R ; v, \theta)) d b \\
=t^{d}(R / d) \boldsymbol{H}^{d-1}(E(k, \varepsilon) \cap \mathrm{S}(p(x), R ; v, \theta)) .
\end{aligned}
$$

Remember that $\varepsilon(R \theta)^{-1} \cdot \mu\left(\mathcal{T}, p(x), \varepsilon(R \theta)^{-1} ; v, \theta\right)$ coincides with

$$
\sum_{k=1}^{\ell} \frac{\boldsymbol{H}^{d}(E(k, t \varepsilon) \cap \operatorname{Cone}(p(x), t R ; v, \theta))}{\boldsymbol{H}^{d}(\operatorname{Cone}(p(x), t R ; v, \theta))}=\sum_{k=1}^{\ell} \frac{\boldsymbol{H}^{d}(E(k, t \varepsilon) \cap \operatorname{Cone}(p(x), t R ; v, \theta))}{t^{d} \boldsymbol{H}^{d}(\operatorname{Cone}(p(x), R ; v, \theta))}
$$

for sufficiently small $t>0$. Therefore, from (12) and (13), we obtain

$$
\begin{aligned}
G\left(\eta^{\prime}, \varepsilon, x\right) & \leq\left(3^{d} \eta^{\prime}\right) \cdot(d / R) \cdot \varepsilon(\theta R)^{-1} \mu\left(\mathcal{T}, p(x), \varepsilon(\theta R)^{-1} ; v, \theta\right) \frac{\boldsymbol{H}^{d}(\operatorname{Cone}(x, R ; v, \theta))}{\boldsymbol{H}^{d}\left(B\left(x, \eta^{\prime}\right)\right)} \\
& \leq(\varepsilon / \eta) \mathcal{M}(\mathcal{T})\left(4 d \cdot 3^{d} \cdot \frac{\boldsymbol{H}^{d}(\operatorname{Cone}(p(x), R ; v, \theta))}{\left(\eta^{\prime}\right)^{d-1} R} \frac{\left(\eta^{\prime}\right)^{d}}{\boldsymbol{H}^{d}\left(B\left(x, \eta^{\prime}\right)\right)}\right) .
\end{aligned}
$$

Here we used the fact that $R \theta \geq \eta^{\prime} / 2 \geq \eta / 4$ and $\varepsilon(R \theta)^{-1} \leq 4 \varepsilon / \eta$. Obviously the term in the last bracket in (14) is bounded by a constant $C(d)$ that depends only on the dimension d. Therefore we have

$$
\frac{\eta}{\varepsilon} G\left(\eta^{\prime}, \varepsilon, x\right) \leq C(d) \mathcal{M}(\mathcal{T})
$$

for any $x \in \mathbb{R}^{d}$ and $0<\varepsilon \leq \eta$. This is nothing but the claim of the proposition. 


\section{A proof of theorem 5}

In this section, we remember the argument in the work of B.Saussol[10] in our special case and prove theorem 5. Let us consider Perron-Frobenius operator

$$
P: \mathcal{L}^{1}(U) \rightarrow \mathcal{L}^{1}(U), \quad f \mapsto P f(x)=\sum_{\mathcal{T}(y)=x} f(y) g(y),
$$

where $\mathcal{L}^{1}(U)$ is the Banach space of integrable functions with $L^{1}$-norm $\|\cdot\|_{\mathcal{L}^{1}}$ and

$$
g(y)=\frac{1}{|\operatorname{det}(D \mathcal{T}(y))|}
$$

Let $\eta$ and $0<\Gamma<1$ be the real number in the statement of theorem 5 . From now on, we regard each element $f \in \mathcal{L}^{1}(U)$ as a function on $\mathbb{R}^{d}$ by setting $f \equiv 0$ on $\mathbb{R}^{d} \backslash U$. For a function $f \in \mathcal{L}^{1}(U)$ and a Borel subset $S \subset \mathbb{R}^{d}$, we define

$$
\operatorname{osc}(f, S)=\operatorname{Esup}_{S} f-\operatorname{Einf}_{S} f
$$

We put $\operatorname{osc}(f, \varnothing)=0$ for convenience. Remark that the function $x \mapsto \operatorname{osc}(f, B(x, \varepsilon))$ is lower semi-continuous and, hence, measurable with respect to $x$. For $\eta>0$, let us put

$$
\operatorname{Osc}(f, \eta)=\sup _{0<\varepsilon \leq \eta} \varepsilon^{-1} \int_{\mathbb{R}^{d}} \operatorname{osc}(f, B(x, \varepsilon)) d \boldsymbol{H}^{d}(x) \in \mathbb{R} \cup\{\infty\}
$$

and consider a space of functions

$$
\mathcal{B V}(U, \eta)=\left\{f \in \mathcal{L}^{1}(U, \mathbb{R}) \mid \operatorname{Osc}(f, \eta)<\infty\right\}
$$

This kind of function spaces are introduced in the paper of Gerhard Keller [8]. The following properties are proved in [8, Theorem 1.13].

Proposition 9 The space of functions $\mathcal{B V}(U, \eta)$ endowed with the norm

$$
\|f\|_{\mathcal{B} \mathcal{V}(U, \eta)}=\operatorname{Osc}(f, \eta)+\|f\|_{\mathcal{L}^{1}}
$$

is a Banach space, which is dense in $\mathcal{L}^{1}(U)$. For any $c>0$, the set of functions

$$
E_{c}=\left\{f \in \mathcal{B} \mathcal{V}(U, \eta) \mid\|f\|_{\mathcal{B V}(U, \eta)} \leq c\right\}
$$

is compact in $\mathcal{L}^{1}(U)$. Moreover, there is a constant $K$ such that, for $f \in \mathcal{B V}(U, \eta)$,

$$
\underset{\mathbb{R}^{d}}{\operatorname{Esup}}|f| \leq K\|f\|_{\mathcal{B V}(U, \eta)}
$$


Theorem 5 is a consequence of the following Lasota-Yorke type inequality.

Lemma 10 Under the assumptions of theorem 5, we have

$$
\operatorname{Osc}(P f, \eta) \leq \Gamma \cdot \operatorname{Osc}(f, \eta)+(\Gamma / \eta)\|f\|_{\mathcal{L}^{1}}
$$

for any non-negative valued functions $f \in \mathcal{B V}(U, \eta)$.

In fact, the set of functions

$$
X=\left\{f \in \mathcal{B} \mathcal{V}(U, \eta) \mid\|f\|_{\mathcal{L}^{1}}=1, f \geq 0 \text { and } \operatorname{Osc}(f, \eta) \leq \Gamma \eta^{-1}(1-\Gamma)^{-1} \cdot\right\}
$$

is a compact convex subset of $\mathcal{L}^{1}(U)$ and $P$-invariant, that is, $P(X) \subset X$. Hence we can get a fixed point $g \in X$ of $P$ as a limit point of the sequence $n^{-1} \sum_{i=0}^{n-1} P^{i}(f)$ for $f \in X$. The measure that has density $g$ with respect to the Lebesgue measure is an absolutely continuous invariant probability measure for $\mathcal{T}$.

Proof of lemma 10 Let us denote $s=\rho(f)^{-1}$ for simplicity. Let $T_{k}: \mathbb{R}^{d} \rightarrow \mathbb{R}^{d}$ be the affine map that coincides with $\mathcal{T}$ on the interior of $U_{k}$ and let $g_{k}=\left|\operatorname{det}\left(D T_{k}\right)\right|^{-1}$. Taking into account that $T_{k}(E(k, \varepsilon))$ is the $\varepsilon$-neighborhood of the boundary $\partial\left(T_{k}\left(U_{k}\right)\right)$, we can see

$$
\begin{aligned}
& \operatorname{osc}(P f, B(x, \varepsilon)) \leq \sum_{k=1}^{\ell} g_{k} \cdot \operatorname{osc}\left(\left(f \circ T_{k}^{-1}\right) \cdot \chi_{T_{k}\left(U_{k}\right)}, B(x, \varepsilon)\right) \\
& \leq \sum_{k=1}^{\ell} g_{k} \cdot \operatorname{osc}\left(f \circ T_{k}^{-1}, B(x, \varepsilon)\right) \cdot \chi_{T_{k}\left(U_{k}\right) \backslash T_{k}(E(k, \varepsilon))}(x) \\
& +\sum_{k=1}^{\ell} g_{k}\left(\operatorname{Esup}_{U_{k} \cap T_{k}^{-1}(B(x, \varepsilon))} f\right) \chi_{T_{k}(E(k, \varepsilon))}(x) \\
& \left.\leq \sum_{k=1}^{\ell} g_{k} \cdot \operatorname{osc}\left(f, B\left(T_{k}^{-1}(x), s \varepsilon\right)\right)\right) \cdot \chi_{U_{k}}\left(T_{k}^{-1}(x)\right) \\
& +\sum_{k=1}^{\ell} g_{k}\left(\operatorname{Esup}_{U_{k} \cap T_{k}^{-1}(B(x, \varepsilon))} f\right) \cdot \chi_{E(k, \varepsilon)}\left(T_{k}^{-1}(x)\right)
\end{aligned}
$$

for $x \in \mathbb{R}^{d}$ and $0<\varepsilon \leq \eta$. We are going to estimate the integrals of the functions

$$
\begin{aligned}
& R_{k}^{(1)}(x)=g_{k} \cdot \operatorname{osc}\left(f, B\left(T_{k}^{-1}(x), s \varepsilon\right)\right) \cdot \chi_{U_{k}}\left(T_{k}^{-1}(x)\right) \quad \text { and } \\
& R_{k}^{(2)}(x)=g_{k} \cdot\left(\operatorname{Esup}_{U_{k} \cap T_{k}^{-1}(B(x, \varepsilon))} f\right) \cdot \chi_{E(k, \varepsilon)}\left(T_{k}^{-1}(x)\right) .
\end{aligned}
$$


By changing the variable $x$ to $y=T_{k}(x)$, we have

$$
\int_{\mathbb{R}^{d}} R_{k}^{(1)}(x) d \boldsymbol{H}^{d}(x) \leq \int_{\mathbb{R}^{d}} \operatorname{osc}(f, B(y, s \varepsilon)) \cdot \chi_{U_{k}}(y) d \boldsymbol{H}^{d}(y) .
$$

Hence we obtain

$$
\int_{\mathbb{R}^{d}} \sum_{k=1}^{\ell} R_{k}^{(1)}(x) d \boldsymbol{H}^{d}(x) \leq \int_{\mathbb{R}^{d}} \mathbf{o s c}(f, B(y, s \varepsilon)) d \boldsymbol{H}^{d}(y) \leq s \varepsilon \cdot \operatorname{Osc}(f, \eta) .
$$

Let us see the second term $R_{k}^{(2)}(x)$. For any point $z \in B\left(T_{k}^{-1}(x),(1-s) \eta\right)$, we have

$$
\left(\operatorname{Esup}_{U_{k} \cap T_{k}^{-1}(B(x, \varepsilon))} f\right) \leq f(z)+\operatorname{osc}(f, B(z, \eta))
$$

because $T_{k}^{-1}(B(x, \varepsilon)) \subset B\left(T_{k}^{-1}(x), s \varepsilon\right)$ is contained in $B(z, \eta)$. Hence

$$
\begin{aligned}
\int_{\mathbb{R}^{d}} \sum_{k=1}^{\ell} R_{k}^{(2)}(x) d \boldsymbol{H}^{d}(x) & \leq \sum_{k=1}^{\ell} \int_{E(k, \varepsilon)} d \boldsymbol{H}^{d}(y) \int_{B(y,(1-s) \eta)} \frac{f(z)+\operatorname{osc}(f, B(z, \eta))}{\boldsymbol{H}^{d}(B(y,(1-s) \eta))} d \boldsymbol{H}^{d}(z) \\
& =\iint_{|y-z| \leq(1-s) \eta} \sum_{k=1}^{\ell} \frac{\chi_{E(k, \varepsilon)}(y)(f(z)+\operatorname{osc}(f, B(z, \eta)))}{\boldsymbol{H}^{d}(B(z,(1-s) \eta))} d \boldsymbol{H}^{2 d}(y, z) \\
& \leq G((1-s) \eta, \varepsilon) \int_{\mathbb{R}^{d}}[f(z)+\operatorname{osc}(f, B(z, \eta))] d \boldsymbol{H}^{d}(z) \\
& \leq G((1-s) \eta, \varepsilon)\|f\|_{\mathcal{L}^{1}}+G((1-s) \eta, \varepsilon) \cdot \eta \cdot \operatorname{Osc}(f, \eta) .
\end{aligned}
$$

Therefore we obtain

$\int_{\mathbb{R}^{d}} \operatorname{osc}(P f, B(x, \varepsilon)) d \boldsymbol{H}^{d}(x) \leq[s+(\eta / \varepsilon) G((1-s) \eta, \varepsilon)] \varepsilon \mathbf{O s c}(f, \eta)+G((1-s) \eta, \varepsilon)\|f\|_{\mathcal{L}^{1}}$ and

$$
\operatorname{Osc}(P f, \eta) \leq(s+\gamma) \operatorname{Osc}(f, \eta)+(\gamma / \eta)\|f\|_{\mathcal{L}^{1}}
$$

where $\gamma=\sup _{0<\varepsilon \leq \eta}\{G((1-s) \eta, \varepsilon)(\eta / \varepsilon)\}$. This obviously implies (15).

\section{Iterations of expanding piecewise linear maps}

Let $(\mathcal{T}, \mathcal{U})$ be an expanding piecewise linear map under our consideration and let $U_{k}$, $1 \leq k \leq d$, be the polyhedra in the associated partition $\mathcal{U}$. As we mentioned before, 
we consider the map $\mathcal{T}$ to be defined only on $U^{\circ}=\cup_{k=1}^{d} \operatorname{int} U_{k}$ and not defined on the complement $U \backslash U^{\circ}$. The iterations of the map $\mathcal{T}$ are again expanding piecewise linear maps. Let us see this fact in detail. For $n \geq 1$, the $n$-th iteration $\mathcal{T}^{n}: U \rightarrow U$ of $\mathcal{T}$ is defined on

$$
U^{\circ}(n)=\left\{x \in U \mid \mathcal{T}^{k}(x) \text { is defined and contained in } U^{\circ} \text { for } 0 \leq k \leq n-1 .\right\}
$$

and not defined on the complement $U \backslash U^{\circ}(n)$. Let $U(n, i), 1 \leq i \leq \ell(n)$, be the closures of connected components of $U^{\circ}(n)$. Then, for each $U(n, i)$, there exists a sequence $\{k(j)\}_{j=0}^{n-1}$ such that

$$
U(n, i)=\cap_{m=0}^{n-1}\left(T_{k(m-1)} \circ T_{k(m-2)} \circ \cdots \circ T_{k(1)} \circ T_{k(0)}\right)^{-1}\left(U_{k(m)}\right) .
$$

Especially $U(n, i)$ is a convex $d$-dimensional polyhedron in $U$ and the restriction of $\mathcal{T}^{n}$ to $U(n, i)$ is $T_{k(m)} \circ T_{k(m-1)} \circ \cdots \circ T_{k(1)} \circ T_{k(0)}$. Therefore the partition $\mathcal{U}(n)=\{U(n, i)\}_{i=1}^{\ell(n)}$ satisfies the conditions (i),(ii) and (iii) in the definition of piecewise linear maps for the iteration $\mathcal{T}^{n}$. Moreover we can see

$$
\rho\left(\mathcal{T}^{n}\right) \geq \rho(\mathcal{T})^{n}
$$

Hence $\left(\mathcal{T}^{n}, \mathcal{U}(n)\right)$ is an expanding piecewise linear map. Theorem 1 follows from

Theorem $11 \lim \inf _{n \rightarrow \infty} \mathcal{M}\left(\mathcal{T}^{n}\right)=0$.

In fact, this theorem and the inequality (16) imply that the conditions in theorem 4 hold for one of the iterations of $\mathcal{T}$. That is, one of the iterations of $\mathcal{T}$ admits an absolutely continuous invariant measure, and so does $\mathcal{T}$ itself.

\section{$6 \quad$ Pseudo-differentials of piecewise linear maps}

In order to estimate the weighted multiplicity of iterations of an expanding piecewise linear map $\mathcal{T}$, we have to look at local properties of them. For this purpose, we introduce a kind of pseudo-differentials of piecewise linear maps. A closed subset $A$ in $\mathbb{R}^{d}$ is said to be $a$ cone in $\mathbb{R}^{d}$ if $v \in A$ implies $t v \in A$ for all $t \geq 0$. A closed subset $A \subset \mathbb{R}^{d}$ is said to be a polyhedral cone in $\mathbb{R}^{d}$ if it is a cone in $\mathbb{R}^{d}$ and a polyhedron at the same time.

Definition A local piecewise linear map is a combination $(T, \mathcal{P})$ of a map $T: A \rightarrow \mathbb{R}^{d}$ on a polyhedral cone $A \subset \mathbb{R}^{d}$ and a family $\mathcal{P}$ of polyhedral cones $A_{k} \subset A, 1 \leq k \leq p$, such that

(i) the interiors of polyhedra $A_{k}$ 's are mutually disjoint,

(ii) $\cup_{k=1}^{p} A_{k}=A$, and

(iii) the restriction of the map $T$ to the interior of each $A_{k}$ is a linear map. 
We will call $T$ itself a local piecewise linear map if the associated partition $\mathcal{P}$ is clear.

The pseudo-differential of a piecewise linear map $\mathcal{T}$ at $x \in U$ is a map $\mathcal{D} \mathcal{T}_{x}: A_{x} \rightarrow \mathbb{R}^{d}$ that is defined on the set

$$
A_{x}=\left\{y \in \mathbb{R}^{d} \mid x+\varepsilon y \in U \text { for sufficiently small } \varepsilon>0\right\}
$$

by

$$
\mathcal{D} \mathcal{T}_{x}(v)=\lim _{\varepsilon \rightarrow+0} \frac{\mathcal{T}(x+2 \varepsilon v)-\mathcal{T}(x+\varepsilon v)}{\varepsilon} .
$$

Remark that the term $(\mathcal{T}(x+2 \varepsilon v)-\mathcal{T}(x+\varepsilon v)) / \varepsilon$ on the right hand side of (17) takes constant value for sufficiently small $\varepsilon>0$.

The partition $\left\{U_{k}\right\}_{k=1}^{\ell}$ associated to the piecewise linear map $\mathcal{T}$ induces a partition of $A_{x}$ into polyhedral cones

$$
P_{x}(\mathcal{T}, k)=\left\{v \in \mathbb{R}^{d} \mid x+\varepsilon v \text { is contained in } U_{k} \text { for sufficiently small } \varepsilon>0 .\right\}
$$

for $1 \leq k \leq \ell$. We denote this partition by

$$
\mathcal{P}_{x}(\mathcal{T})=\left\{P_{x}(\mathcal{T}, k) ; 1 \leq k \leq \ell\right\} .
$$

Remark (1) Some of the elements in the partition $\mathcal{P}_{x}(\mathcal{T})$ may be empty or have empty interior. But we count them as an element of the partition for the consistency of the index $k$. In the argument below, we will ignore such elements. (2) We consider that $\mathcal{D} \mathcal{T}_{x}$ is defined only on the union of the interiors of $P_{x}(\mathcal{T}, k), 1 \leq k \leq \ell$, and not defined on the complement.

The combination $\left(\mathcal{D} \mathcal{T}_{x}, \mathcal{P}_{x}(\mathcal{T})\right)$ is a local piecewise linear map on $A_{x}$. Notice that there is only finitely many distinct maps among the pseudo-differentials $\mathcal{D} \mathcal{T}_{x}$ for $x \in U$. This fact is important for our argument below.

If $x \in U^{\circ}=\cup_{k} \operatorname{int}\left(U_{k}\right)$, the pseudo-differential $\mathcal{D} \mathcal{T}_{x}$ is a linear map and that is nothing but the ordinary differential of $\mathcal{T}$ at $x$. If $x \in U \backslash U^{\circ}$, the map $\mathcal{D} \mathcal{T}_{x}$ is not a linear map, but a local piecewise linear map. Especially, if $x$ is on the boundary of $U$, the domain $A_{x}$ is a proper subset of $\mathbb{R}^{d}$. For a local piecewise linear map $\mathcal{S}: A \rightarrow \mathbb{R}^{p}$, we define its pseudo-differential $\mathcal{D} \mathcal{S}_{x}$ at a point $x \in A$ in the exactly same manner.

Now we are going to define second order pseudo-differentials of the map $\mathcal{T}$ as a pseudodifferential of $\mathcal{D} \mathcal{T}_{x}$. They are quite different from the ordinary second order differentials. For a point $x \in U$ and $v \in A_{x}$, the second order pseudo-differential of a piecewise linear map $\mathcal{T}$ at $(x, v)$ is a map $\mathcal{D}^{2} \mathcal{T}_{x, v}: A_{x, v} \rightarrow \mathbb{R}^{d}$ that is defined on the set

$$
\begin{aligned}
A_{x, v} & =\left\{y \in \mathbb{R}^{d} \mid y \in A_{x+\varepsilon v} \text { for sufficiently small } \varepsilon>0 .\right\} \\
& =\left\{y \in \mathbb{R}^{d} \mid x+\varepsilon v+\varepsilon^{2} y \in U \text { for sufficiently small } \varepsilon>0 .\right\}
\end{aligned}
$$


by

$$
\begin{aligned}
\mathcal{D}^{2} \mathcal{T}_{x, v}(w) & =\lim _{\varepsilon \rightarrow+0} \mathcal{D}\left(\mathcal{D} \mathcal{T}_{x}\right)_{\varepsilon v}(w) \\
& =\lim _{\varepsilon \rightarrow+0} \frac{\mathcal{T}\left(x+\varepsilon v+2 \varepsilon^{2} w\right)-\mathcal{T}\left(x+\varepsilon v+\varepsilon^{2} w\right)}{\varepsilon^{2}}
\end{aligned}
$$

Remark again that the fraction on the right hand side of (18) takes a constant value for sufficiently small $\varepsilon>0$. The partition $\mathcal{P}_{x}(\mathcal{T})$ induces a partition of $A_{x, v}$,

$$
\mathcal{P}_{x, v}(\mathcal{T})=\left\{P_{x, v}(\mathcal{T}, k) \mid 1 \leq i \leq \ell\right\}
$$

where

$$
\begin{aligned}
P_{x, v}(\mathcal{T}, k) & =\left\{w \in \mathbb{R}^{d} \mid w \in P_{x+\varepsilon v}(\mathcal{T}, k) \text { for sufficiently small } \varepsilon>0\right\} \\
& =\left\{w \in \mathbb{R}^{d} \mid x+\varepsilon v+\varepsilon^{2} w \in U_{k} \text { for sufficiently small } \varepsilon>0\right\} .
\end{aligned}
$$

Then the combination $\left(\mathcal{D}^{2} \mathcal{T}_{x, v}, \mathcal{P}_{x, v}(\mathcal{T})\right)$ is a local piecewise linear map. If $x \in U^{\circ}$, we have $\mathcal{D}^{2} \mathcal{T}_{x, v}=\mathcal{D} \mathcal{T}_{x}$ for any $v \neq 0$. If $x \notin U^{\circ}$ and $v$ is contained in the interior of $P_{x}(\mathcal{T}, k)$ for some $1 \leq k \leq \ell$, the second order pseudo-differential $\mathcal{D}^{2} \mathcal{T}_{x, v}$ is the linear map that coincides with $\mathcal{D} \mathcal{T}_{x}$ on a neighborhood of $v$. Otherwise $\mathcal{D}^{2} \mathcal{T}_{x, v}$ is not a linear map while it is still a local piecewise linear map.

For $m \geq 2$, we define pseudo-differentials of order $m$ of a piecewise linear map $\mathcal{T}$ in the following inductive manner. Let $\mathcal{I}_{m}$ be the set of combinations of a point $x \in U$ and vectors $0 \neq v(i) \in \mathbb{R}^{d}, 1 \leq i \leq m-1$, such that $v(i) \in A_{(x, v(1), \ldots, v(i-1))}$ for $1 \leq i \leq m-1$. For $\boldsymbol{v}=(x, v(1), v(2), \ldots, v(m-1)) \in \mathcal{I}_{m}$, the pseudo-differential of $\mathcal{T}$ of order $m$ at $\boldsymbol{v}$ is a map $\mathcal{D}^{m} \mathcal{T}_{v}: A_{v} \rightarrow \mathbb{R}^{d}$ that is defined on the set

$$
A_{v}=\left\{w \in \mathbb{R}^{d} \mid x+\sum_{i=1}^{m-1} \varepsilon^{i} v(i)+\varepsilon^{m} w \in U \text { for sufficiently small } \varepsilon>0 .\right\}
$$

by

$$
\begin{aligned}
\mathcal{D}^{m} \mathcal{T}_{\boldsymbol{v}}(w) & =\lim _{\varepsilon \rightarrow+0} \mathcal{D}\left(\mathcal{D}^{m-1} \mathcal{T}_{\boldsymbol{v}^{\prime}}\right)_{\varepsilon \cdot v(m-1)}(w) \\
& =\lim _{\varepsilon \rightarrow+0} \frac{\mathcal{T}\left(x+\sum_{i=1}^{m-1} \varepsilon^{i} v(i)+2 \varepsilon^{m} w\right)-\mathcal{T}\left(x+\sum_{i=1}^{m-1} \varepsilon^{i} v(i)+\varepsilon^{m} w\right)}{\varepsilon^{m}}
\end{aligned}
$$

where $\boldsymbol{v}^{\prime}=(x, v(1), v(2), \ldots, v(m-2)) \in \mathcal{I}_{m-1}$. Let

$$
\mathcal{P}_{\boldsymbol{v}}(\mathcal{T})=\left\{P_{v}(\mathcal{T}, k) \mid 1 \leq k \leq \ell\right\}
$$

be a partition of $A_{v}$ defined by

$$
P_{\boldsymbol{v}}(\mathcal{T}, k)=\left\{w \in \mathbb{R}^{d} \mid x+\sum_{i=1}^{m-1} \varepsilon^{i} v(i)+\varepsilon^{m} w \in U_{k} \text { for sufficiently small } \varepsilon>0 .\right\} .
$$


Then the combination $\left(\mathcal{D}^{m} \mathcal{T}_{\boldsymbol{v}}, \mathcal{P}_{\boldsymbol{v}}(\mathcal{T})\right)$ is a local piecewise linear map.

From the definition (19), the pseudo-differentials $\mathcal{D}^{m} \mathcal{T}_{\boldsymbol{v}}$ of the map $\mathcal{T}$ of order $m>1$ coincide with one of pseudo-differentials of order $m-1$ of $\mathcal{T}$. Let $\mathcal{I}_{m}^{\circ}$ be the subset of $\mathcal{I}^{m}$ that consists of elements $\boldsymbol{v}=(x, v(1), v(2), \ldots, v(m-1))$ for which the set of vectors $v(1), v(2), \ldots, v(m-1)$ are linearly independent. Let $\boldsymbol{D}_{m}(\mathcal{T})$ be the set of distinct maps among $\mathcal{D}^{m} \mathcal{T}_{\boldsymbol{v}}, \boldsymbol{v} \in \mathcal{I}_{m}^{\circ}$. Then we have

$$
D_{1}(\mathcal{T}) \supset D_{2}(\mathcal{T}) \supset D_{3}(\mathcal{T}) \supset \cdots \supset D_{d-1}(\mathcal{T}) \supset D_{d}(\mathcal{T})
$$

Definition Let $L$ be a linear subspace in $\mathbb{R}^{d}$. A subset $B \subset \mathbb{R}^{d}$ is said to be $L$-cylindrical if $y+w \in B$ for any $y \in B$ and any $w \in L$.

For $v \in \mathcal{I}_{m}^{\circ}$, let $L_{v}$ be the $(m-1)$-dimensional subspace of $\mathbb{R}^{d}$ spanned by the vectors $v(1), v(2), \ldots, v(m-1)$. Then each element $P=P_{\boldsymbol{v}}(\mathcal{T}, k)$ in the partition $\mathcal{P}_{\boldsymbol{v}}(\mathcal{T})$ is $L_{\boldsymbol{v}^{-}}$ cylindrical. Especially, $P=L_{v}=\mathbb{R}^{d}$ for $v \in \mathcal{I}_{d}^{\circ}$ and, hence, the pseudo-differentials $\mathcal{D}^{d} \mathcal{T}_{v}$ of order $d$ are linear maps. So $D_{d}(\mathcal{T})$ consists of linear maps in $D_{1}(\mathcal{T})$.

\section{An elementary estimate}

Before going into the main part of the proof of theorem 11, we prepare an elementary estimate for volume of $\varepsilon$-neighborhoods of cones in $\mathbb{R}^{p}$. For $0 \leq r<r^{\prime}$, let us denote

$$
B^{p}(r)=\left\{y \in \mathbb{R}^{p} \mid\|y\|<r\right\}
$$

and

$$
\operatorname{Shell}^{p}\left(r, r^{\prime}\right)=\left\{y \in \mathbb{R}^{p} \mid r \leq\|y\| \leq r^{\prime}\right\} .
$$

Let $A \subset \mathbb{R}^{p}$ be a cone in $\mathbb{R}^{p}$. (See the beginning of section 6 for the definition.) For $\varepsilon>0$, let $N(A, \varepsilon)$ be the $\varepsilon$-neighborhood of $A$, that is,

$$
N(A, \varepsilon)=\left\{y \in \mathbb{R}^{p} \mid d(y, A)<\varepsilon\right\} .
$$

In section 8 , we will use the following proposition.

Proposition 12 Let $\Psi: \mathbb{R}^{p} \rightarrow \mathbb{R}^{p}$ be a linear map and let $\varepsilon>0$. If

$$
\inf _{v \in \mathbb{R}^{p} \backslash\{0\}}\|\Psi(v)\| /\|v\| \geq 12 \varepsilon
$$

and $A \neq\{0\}$, we have

$$
\boldsymbol{H}^{p}\left(\Psi^{-1}(N(A, \varepsilon)) \cap B^{p}(1)\right) \leq 3 \cdot \boldsymbol{H}^{p}\left(\Psi^{-1}(N(A, \varepsilon)) \cap \text { Shell }^{p}(1 / 3,1)\right) .
$$


Proof. For a cone $A \neq\{0\}$ in $\mathbb{R}^{d}$, we can take a sequence of cones $A_{i}$ in $\mathbb{R}^{d}$, each of which consists of finitely many half-lines, so that $A_{i} \cap B^{p}(1)$ converges to $A \cap B^{p}(1)$ in Hausdorff distance. Thus it is enough to prove the claim in the case where the cone $A$ consists of finitely many half-lines. We assume this below. For each $x \in \mathbb{R}^{p}$, let $\sigma(x)$ be a point in $A$ such that $d(x, y)=d(x, A) \equiv \inf _{y \in A} d(x, y)$. Notice that $\sigma(x)$ is uniquely determined for all $x \in \mathbb{R}^{p}$ but for points on finitely many hyperplanes.

Lemma 13 For a map $F: \mathbb{R}^{p} \rightarrow \mathbb{R}^{p}$ defined by $F(x)=x+\sigma(x)$ and $\varepsilon>0$, we have

(1) $F$ is injective $\boldsymbol{H}^{p}$-almost everywhere,

(2) $\sigma(F(x))=2 \sigma(x)$ for $\boldsymbol{H}^{p}$-almost every $x \in \mathbb{R}^{p}$,

(3) $|\operatorname{det} D F(x)|=2$ for $H^{p}$-almost every point $x$ on $N(A, \varepsilon) \backslash B^{p}(\varepsilon)$.

Proof. Let us show (1). We prove that, if $\sigma(x)$ and $\sigma(y)$ are uniquely determined for points $x$ and $y$ and if $F(y)=F(x)$, we have $x=y$. From the symmetry on $x$ and $y$, we can assume $\|x-\sigma(x)\| \leq\|y-\sigma(y)\|$. Then, if $F(x)=F(y)$, we have

$$
\begin{aligned}
2\|y-\sigma(x)\| & \leq\|2 y-F(x)\|+\|F(x)-2 \sigma(x)\|=\|2 y-F(y)\|+\|F(x)-2 \sigma(x)\| \\
& \leq\|y-\sigma(y)\|+\|x-\sigma(x)\| \leq 2\|y-\sigma(y)\| .
\end{aligned}
$$

This implies $\sigma(x)=\sigma(y)$. Hence $x=-\sigma(x)+F(x)=-\sigma(y)+F(y)=y$.

Let us show (2). Since $2 \sigma(x) \in A$, we have

$$
\|\sigma(F(x))-F(x)\| \leq\|2 \sigma(x)-F(x)\|=\|\sigma(x)-x\| .
$$

Hence

$$
\|(\sigma(F(x)) / 2)-x\| \leq\|\sigma(F(x))-F(x)\| / 2+\|F(x)-2 x\| / 2 \leq\|\sigma(x)-x\| .
$$

This implies $\sigma(F(x))=2 \sigma(x)$ if $\sigma(x)$ is unique.

Let us show (3). For almost every point $x \in N(A, \varepsilon) \backslash B^{p}(\varepsilon)$, there exists a neighborhood on which the map $\sigma$ coincides with the orthogonal projection to one of the half-lines in $A$. Then it is easy to see that the determinant of the map $F$ at $x$ equals to 2 .

Since the map $\Psi$ is linear, the claim of proposition 12 is equivalent to the inequality

$$
\boldsymbol{H}^{p}\left(N(A, \varepsilon) \cap \Psi\left(B^{p}(1)\right)\right) \leq 3 \cdot \boldsymbol{H}^{p}\left(N(A, \varepsilon) \cap \Psi\left(\text { Shell }^{p}(1 / 3,1)\right)\right) .
$$

For $i \geq 0$, let us put

$$
\Gamma_{i}=\left\{y \in N(A, \varepsilon) \mid \sigma(y) \in \Psi\left(\operatorname{Shell}^{p}\left(2^{-i-1}(5 / 6), 2^{-i}(5 / 6)\right)\right)\right\} .
$$

Notice that, if $x \in N(A, \varepsilon) \backslash B^{p}(\varepsilon)$, the point $\sigma(x)$ is not the origin 0 and the angle between the vectors $x$ and $\sigma(x)$ is smaller that $\pi / 2$. So we have $\|F(x)\| \geq\|x\| \geq \varepsilon$ for 
$x \in N(A, \varepsilon) \backslash B^{p}(\varepsilon)$. From the claim (2) of lemma 13, we can see that $\|\sigma(F(x))\|=2\|\sigma(x)\|$ and $\|F(x)-\sigma(F(x))\|=\|x-\sigma(x)\|<\varepsilon$ for $\boldsymbol{H}^{p}$-a.e. $x \in N(A, \varepsilon)$. Hence we have

$$
F\left(\Gamma_{i} \backslash B^{p}(\varepsilon)\right) \subset \Gamma_{i-1} \backslash B^{p}(\varepsilon) \quad \boldsymbol{H}^{p} \text {-a.e. }
$$

From claims (1) and (3) of lemma 13, we obtain

$$
\boldsymbol{H}^{p}\left(\Gamma_{i} \backslash B^{p}(\varepsilon)\right) \leq(1 / 2) \cdot \boldsymbol{H}^{p}\left(\Gamma_{i-1} \backslash B^{p}(\varepsilon)\right) .
$$

Therefore

$$
\boldsymbol{H}^{p}\left(\cup_{i=1}^{\infty} \Gamma_{i} \backslash B^{p}(\varepsilon)\right)=\sum_{i=1}^{\infty} \boldsymbol{H}^{p}\left(\Gamma_{i} \backslash B^{p}(\varepsilon)\right) \leq \sum_{i=1}^{\infty} 2^{-i} \cdot \boldsymbol{H}^{p}\left(\Gamma_{0}\right)=\boldsymbol{H}^{p}\left(\Gamma_{0}\right) .
$$

For $x \in N(A, \varepsilon) \cap \Psi\left(B^{p}(1 / 3)\right)$, we have

$$
\left\|\Psi^{-1}(\sigma(x))\right\| \leq\left\|\Psi^{-1}(x)\right\|+\left\|\Psi^{-1}(x-\sigma(x))\right\|<(1 / 3)+(12 \varepsilon)^{-1} \varepsilon=5 / 12 .
$$

So $N(A, \varepsilon) \cap \Psi\left(B^{p}(1 / 3)\right)$ is contained in $\cup_{i=1}^{\infty} \Gamma_{i}$, and

$$
\boldsymbol{H}^{p}\left(N(A, \varepsilon) \cap \Psi\left(B^{p}(1 / 3)\right) \backslash B^{p}(\varepsilon)\right) \leq \boldsymbol{H}^{p}\left(\cup_{i=1}^{\infty} \Gamma_{i} \backslash B^{p}(\varepsilon)\right) \leq \boldsymbol{H}^{p}\left(\Gamma_{0}\right) .
$$

We have $1 / 3 \leq\left\|\Psi^{-1}(x)\right\| \leq 1$ for $x \in \Gamma_{0}$, because $5 / 12 \leq \Psi^{-1}(\sigma(x)) \leq 5 / 6$ and

$$
\left\|\Psi^{-1}(x)-\Psi^{-1}(\sigma(x))\right\| \leq(12 \varepsilon)^{-1} \varepsilon \leq 1 / 12 .
$$

Thus $\Gamma_{0}$ is contained in $N(A, \varepsilon) \cap \Psi\left(\right.$ Shell $\left.^{p}(1 / 3,1)\right)$. Therefore

$$
\boldsymbol{H}^{p}\left(N(A, \varepsilon) \cap \Psi\left(B^{p}(1 / 3)\right) \backslash B^{p}(\varepsilon)\right) \leq \boldsymbol{H}^{p}\left(N(A, \varepsilon) \cap \Psi\left(\operatorname{Shell}^{p}(1 / 3,1)\right)\right) .
$$

On the other hand, we have

$$
\boldsymbol{H}^{p}\left(B^{p}(\varepsilon)\right) \leq \boldsymbol{H}^{p}\left(N(A, \varepsilon) \cap \Psi\left(\operatorname{Shell}^{p}(1 / 3,1)\right)\right),
$$

because $N(A, \varepsilon) \cap \Psi\left(\right.$ Shell $\left.{ }^{p}(1 / 3,1)\right)$ contains at least one ball with radius $\varepsilon$ when $A \neq\{0\}$. The inequalities (21) and (22) imply (20). We proved proposition 12.

\section{Proof of theorem 11}

In this section we prove theorem 11 and complete the proof of theorem 1. Let us assume

$$
\rho(\mathcal{T})>48 \pi
$$


replacing $\mathcal{T}$ by its iteration if necessary. Let us prepare some notations. Consider an element $\boldsymbol{v}=(x, v(1), v(2), \ldots, v(m-1))$ of $\mathcal{I}_{m}^{\circ}$. As in section 6 , we denote the $(m-1)$ dimensional subspace of $\mathbb{R}^{d}$ spanned by vectors $v(i), 1 \leq i \leq m-1$, by $L_{v}$. Let $L_{v}^{\frac{1}{}}$ be the orthogonal complement of $L_{\boldsymbol{v}}$ in $\mathbb{R}^{d}$ and let $\pi_{v}: \mathbb{R}^{d} \rightarrow L_{v}^{\perp}$ be the orthogonal projection.

For $x \in L_{v}^{\perp}$ and $r>0$, we put

$$
B_{\boldsymbol{v}}(x, r)=\left\{y \in L_{\boldsymbol{v}}^{\perp} \mid\|y-x\| \leq r\right\} .
$$

For $v \in L_{v}^{\perp} \backslash\{0\}$ and $0 \leq \theta \leq \pi$, we put

$$
\text { Cone }_{\boldsymbol{v}}(v, \theta)=\left\{y \in L_{\boldsymbol{v}}^{\perp} \backslash\{0\} \mid \angle(y, v)<\theta\right\} .
$$

Then, for $1 \leq k \leq \ell$, we define

$$
P_{v}(\mathcal{T}, k ; v, \theta)=P_{v}(\mathcal{T}, k) \cap \pi_{v}^{-1}\left(\operatorname{Cone}_{v}(v, \theta)\right) .
$$

Notice that elements $P_{\boldsymbol{v}}(\mathcal{T}, k)$ of the partition $\mathcal{P}_{\boldsymbol{v}}(\mathcal{T})$ and $P_{\boldsymbol{v}}(\mathcal{T}, k ; v, \theta)$ are $L_{\boldsymbol{v}}$-cylindrical

We want to define weighted multiplicity for each pseudo-differential $\mathcal{D}^{m} \mathcal{T}_{\boldsymbol{v}}$ at $\boldsymbol{v} \in \mathcal{I}_{m}^{\circ}$ of order $1 \leq m \leq d-1$. For $1 \leq k \leq \ell, \varepsilon>0, v \in L_{\boldsymbol{v}}^{\perp} \backslash\{0\}$ and $0<\theta \leq \pi$, we put

$$
E_{\boldsymbol{v}}(\mathcal{T}, k, \varepsilon ; v, \theta)=\left\{y \in \mathbb{R}^{d} \mid d\left(T_{\boldsymbol{v}, k}(y), T_{\boldsymbol{v}, k}(z)\right)<\varepsilon \text { for some } z \in \partial P_{\boldsymbol{v}}(\mathcal{T}, k ; v, \theta) .\right\}
$$

where $\mathcal{T}_{\boldsymbol{v}, k}: \mathbb{R}^{d} \rightarrow \mathbb{R}^{d}$ is the linear map that coincides with $\mathcal{D}^{m} \mathcal{T}_{\boldsymbol{v}}$ on the interior of $P_{\boldsymbol{v}}(\mathcal{T}, k)$. Since this definition does not depend on $v$ when $\theta=\pi$, we will write it simply

$$
E_{v}(\mathcal{T}, k, \delta)=E_{v}(\mathcal{T}, k, \delta ; v, \pi)
$$

Notice that these subsets are also $L_{v^{-}}$cylindrical.

For $v \in L_{v}^{\perp} \backslash\{0\}, 0 \leq \theta \leq \pi$ and $\varepsilon>0$, put

$$
\boldsymbol{m}_{\boldsymbol{v}}(\mathcal{T}, \varepsilon ; v, \theta)=\varepsilon^{-1} \sum_{k=1}^{\ell} \frac{\boldsymbol{H}^{d-m+1}\left(E_{\boldsymbol{v}}(\mathcal{T}, k, \varepsilon \theta ; v, \theta) \cap B_{\boldsymbol{v}}(0,1)\right)}{\boldsymbol{H}^{d-m+1}\left(\mathrm{Cone}_{\boldsymbol{v}}(v, \theta) \cap B_{\boldsymbol{v}}(0,1)\right)}
$$

In the case of first order pseudo-differentials, or in the case $m=1$ and $v=(x)$, we have

$$
\mu(\mathcal{T}, x, \varepsilon ; v, \theta) \leq m_{v}(\mathcal{T}, \varepsilon ; v, \theta) .
$$

( (24) is not an equality but only an inequality because $\boldsymbol{m}_{\boldsymbol{v}}(\mathcal{T}, \varepsilon ; v, \theta)$ counts the neighborhood of the boundary of $\mathrm{Cone}_{v}(v, \theta)$.)

We define the weighted multiplicity of the pseudo-differential $\mathcal{D}^{m} \mathcal{T}_{v}, v \in \mathcal{I}_{m}^{\circ}$, by

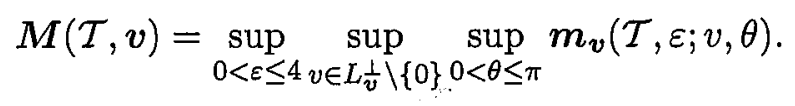


Then we put, for $1 \leq m \leq d-1$,

$$
M_{m}(\mathcal{T})=\sup _{\boldsymbol{v} \in \mathcal{I}_{m}^{\circ}} M(\mathcal{T}, v)
$$

Remark that the supremum is taken over $\mathcal{I}_{m}^{\circ}$ and not on $\mathcal{I}_{m}$. It is not difficult to check that $\boldsymbol{M}_{m}(\mathcal{T})$ is finite for $1 \leq m \leq d-1$. From (24), we have

$$
\mathcal{M}(\mathcal{T}) \leq M_{1}(\mathcal{T})
$$

For these weighted multiplicity, we will prove the following claim.

Proposition 14 There exist constants $K(d)$, which depends only on the dimension $d$, and $N(\mathcal{T})$, which depends on the map $\mathcal{T}$, such that

$$
M_{m}\left(\mathcal{T}^{n+1}\right)<3\left[s+K(d) M_{m+1}(\mathcal{T})\right] M_{m}\left(\mathcal{T}^{n}\right)+3 K(d) M_{m+1}(\mathcal{T})
$$

for $1 \leq m \leq d-2$ and $n \geq N(\mathcal{T})$, where $s=\rho(\mathcal{T})^{-1}$.

Before going into the proof of this proposition, we show that theorem 11 follows from proposition 14. Remark that each map $\mathcal{D}^{d-1} \mathcal{T}_{u}^{n}$ for $u \in \mathcal{I}_{d-1}^{\circ}$ is a linear map or a local piecewise linear map whose associated partition is a partition of $\mathbb{R}^{d}$ by the hyperplane $L_{\boldsymbol{v}}$. Moreover, its expansion rate is not smaller than $\rho(\mathcal{T})^{n}$. Hence we can see

$$
\boldsymbol{m}_{\boldsymbol{v}}(\mathcal{T}, \varepsilon ; v, \theta) \leq 2 \cdot \varepsilon \theta \cdot \rho\left(\mathcal{T}^{n}\right)^{-1} \leq 8 \pi \rho(\mathcal{T})^{-n}
$$

for any $\boldsymbol{v} \in \mathcal{I}_{d-1}^{\circ}, v \in L_{\boldsymbol{v}}^{\perp} \backslash\{0\}, 0<\varepsilon \leq 4$ and $0<\theta \leq \pi$. Hence

$$
\lim _{n \rightarrow \infty} M_{d-1}\left(\mathcal{T}^{n}\right)=0
$$

Assume that we have proved

$$
\liminf _{n \rightarrow \infty} M_{m+1}\left(\mathcal{T}^{n}\right)=0
$$

for some $1 \leq m \leq d-2$. Then, for any $\Delta>0$, we can find arbitrary large $k>0$ such that $M_{m+1}\left(\mathcal{T}^{k}\right)<\Delta$. Applying proposition 14 to $\mathcal{T}^{k}$, we have

$$
M_{m}\left(\mathcal{T}^{(n+1) k}\right)<3\left\{s^{k}+\Delta \cdot K(d)\right\} M_{m}\left(\mathcal{T}^{n k}\right)+3 \Delta \cdot K(d)
$$

for sufficiently large $n$. If $3\left(s^{k}+\Delta \cdot K(d)\right)<1$, this implies

$$
\liminf _{n \rightarrow \infty} M_{m}\left(\mathcal{T}^{n}\right) \leq \frac{3 \Delta \cdot K(d)}{1-3\left(s^{k}+\Delta \cdot K(d)\right)} .
$$

Since we can take $\Delta>0$ arbitrary small and $k$ arbitrary large, we obtain

$$
\liminf _{n \rightarrow \infty} M_{m}\left(\mathcal{T}^{n}\right)=0
$$


Therefore, by induction on $m$, we obtain

$$
\liminf _{n \rightarrow \infty} M_{1}\left(\mathcal{T}^{n}\right)=0 .
$$

From (25), this implies theorem 11.

Remark We can prove that $M_{m}\left(\mathcal{T}^{n}\right), 1 \leq m \leq d-1$, converges to 0 exponentially fast by slightly modifying the above argument.

Now our task become proving proposition 14 . We first give a consequence of proposition 12 in the last section. Put

$$
\operatorname{Shell}_{v}=\left\{y \in L_{\boldsymbol{v}}^{\perp} \mid 1 / 3 \leq\|y\| \leq 1\right\}
$$

and

$$
\tilde{\boldsymbol{m}}_{v}(\mathcal{T}, \varepsilon ; v, \theta)=\varepsilon^{-1} \sum_{k=1}^{\ell} \frac{\boldsymbol{H}^{d-m+1}\left(E_{v}(\mathcal{T}, k, \varepsilon \theta ; v, \theta) \cap \mathrm{Shell}_{\boldsymbol{v}}\right)}{\boldsymbol{H}^{d-m+1}\left(\operatorname{Cone}_{v}(v, \theta) \cap B_{\boldsymbol{v}}(0,1)\right)}
$$

Then we have the following inequality as a consequence of proposition 12 .

Lemma 15 For any $0<\varepsilon \leq 4, v \in L_{v}^{\perp} \backslash\{0\}$ and $0<\theta \leq \pi$, we have

$$
\boldsymbol{m}_{\boldsymbol{v}}(\mathcal{T}, \varepsilon ; v, \theta) \leq 3 \tilde{\boldsymbol{m}}_{\boldsymbol{v}}(\mathcal{T}, \varepsilon ; v, \theta) .
$$

Proof. Let $T_{\boldsymbol{v}, k}$ be the linear map that coincides with $\mathcal{D}^{m} \mathcal{T}_{\boldsymbol{v}}$ on the interior of $P_{\boldsymbol{v}}(\mathcal{T}, k)$ as before. Let $\pi: \mathbb{R}^{d} \rightarrow T_{v, k}\left(L_{v}\right)^{\perp}$ be the orthogonal projection to the normal complement of $T_{\boldsymbol{v}, k}\left(L_{\boldsymbol{v}}\right)$. Put $\tau=\pi \circ T_{\boldsymbol{v}, k}$ and $A=\tau\left(\partial P_{\boldsymbol{v}}(\mathcal{T}, k ; v, \theta)\right)$. Then, since $T_{\boldsymbol{v}, k}\left(\partial P_{\boldsymbol{v}}(\mathcal{T}, k ; v, \theta)\right)$ is $T_{\boldsymbol{v}, k}\left(L_{\boldsymbol{v}}\right)$-cylindrical, we have

$$
E_{\boldsymbol{v}}(\mathcal{T}, k, \varepsilon ; v, \theta) \cap B_{v}(0,1)=\left\{y \in B_{\boldsymbol{v}}(0,1) \mid d(\tau(y), A)<\varepsilon \theta\right\}
$$

Since $\rho(\mathcal{T})>48 \pi \geq 12 \varepsilon \theta$, we can apply proposition 12 for $\Psi=\tau$ and $A \subset T_{\boldsymbol{v}, k}\left(L_{\boldsymbol{v}}\right)^{\perp}$, and get

$$
\boldsymbol{H}^{d-m+1}\left(E_{\boldsymbol{v}}(\mathcal{T}, k, \varepsilon \theta ; v, \theta) \cap B_{\boldsymbol{v}}(0,1)\right) \leq 3 \boldsymbol{H}^{d-m+1}\left(E_{\boldsymbol{v}}(\mathcal{T}, k, \varepsilon \theta ; v, \theta) \cap \text { Shell }_{\boldsymbol{v}}\right) .
$$

This obviously implies the lemma.

Let us fix $v=(\alpha, v(1), v(2), \ldots, v(m-1)) \in \mathcal{I}_{m}^{\circ}, v \in L_{v}^{\perp} \backslash\{0\}, 0<\theta \leq \pi$ and $n>0$. We will consider a fixed element $P_{v}\left(\mathcal{T}^{n}, k\right)$ in the partition $\mathcal{P}_{\boldsymbol{v}}\left(\mathcal{T}^{n}\right)$ from now on until the last part of the proof. Thus we put $Q=P_{v}\left(\mathcal{T}^{n}, k ; v, \theta\right)$ for simplicity of notation. Notice that the partition $\mathcal{P}_{\boldsymbol{v}}\left(\mathcal{T}^{n+1}\right)$ subdivides $\mathcal{P}_{\boldsymbol{v}}\left(\mathcal{T}^{n}\right)$. By changing the index, we assume that

$$
Q(i):=P_{v}\left(\mathcal{T}^{n+1}, i\right) \quad 1 \leq i \leq q
$$


are the elements of the partition $\mathcal{P}_{\boldsymbol{v}}\left(\mathcal{T}^{n+1}\right)$ that intersect $Q$. Then we have $\cup_{i=1}^{q} Q(i)=Q$. Let $T$ be the linear map that coincides with $\mathcal{D}^{m} \mathcal{T}_{v}^{n}$ on the interior of $Q$ and put

$$
\boldsymbol{w}=\left(\lim _{\varepsilon \rightarrow+0} \mathcal{T}^{n}\left(\alpha+\sum_{j=1}^{m-1} \varepsilon^{j} v(j)+\varepsilon^{m} w\right), T(v(1)), T(v(2)), \ldots, T(v(m-1))\right)
$$

where $w$ is an arbitrary element in the interior of $P_{\boldsymbol{v}}\left(\mathcal{T}^{n}, k\right)$. Then, again, by changing the index for the elements in $\mathcal{P}_{v}(\mathcal{T})$, we can assume

$$
Q(i)=Q \cap T^{-1}\left(P_{w}(\mathcal{T}, i)\right) \quad \text { for } 1 \leq i \leq q .
$$

For $1 \leq i \leq q$, let $S_{i}$ be the linear map that coincides with $\mathcal{D}^{m} \mathcal{T}_{w}$ on the interior of $P_{w}(\mathcal{T}, i)$. Then we have $\mathcal{D}^{m} \mathcal{T}_{v}^{n+1}=S_{i} \circ T$ on $Q(i)$.

Now we want to estimate the sum

$$
\sum_{i=1}^{q} \boldsymbol{H}^{d-m+1}\left(E_{\boldsymbol{v}}\left(\mathcal{T}^{n+1}, i, \varepsilon \theta ; v, \theta\right) \cap \operatorname{Shell}_{v}\right) .
$$

By the notation we prepared above, we can write, for $\delta>0$,

$$
E_{\boldsymbol{v}}\left(\mathcal{T}^{n+1}, i, \delta ; v, \theta\right)=\left\{y \in \mathbb{R}^{d} \mid d\left(S_{i} \circ T(y), S_{i} \circ T(\partial Q(i))\right)<\delta\right\}
$$

and

$$
E_{v}\left(\mathcal{T}^{n}, k, \delta ; v, \theta\right)=\left\{w \in \mathbb{R}^{d} \mid d(T(w), T(\partial Q))<\delta\right\} .
$$

We divide the subset $E_{v}\left(\mathcal{T}^{n+1}, i, \varepsilon \theta ; v, \theta\right) \cap \operatorname{Shell}_{v}$ in two parts. Remember that, for each point $y \in E_{v}\left(\mathcal{T}^{n+1}, i, \varepsilon \theta ; v, \theta\right)$, the image $S_{i} \circ T(y)$ is contained in the $\varepsilon \theta$-neighborhood of the boundary $S_{i} \circ T(\partial Q(i))$. On the other hand, the boundary $S_{i} \circ T(\partial Q(i))$ is contained in the union of $S_{i}(T(\partial Q))$ and $S_{i}\left(\partial P_{w}(\mathcal{T}, i)\right)$ from (26). Thus we define $R_{1}(\varepsilon, i)$ as a subset of $E_{v}\left(\mathcal{T}^{n+1}, i, \varepsilon \theta ; v, \theta\right) \cap$ Shell $_{\boldsymbol{v}}$ that consists of points $y$ in $E_{v}\left(\mathcal{T}^{n}, k, \varepsilon \theta ; v, \theta\right) \cap$ Shell $_{v}$ such that $S_{i} \circ T(y)$ is contained in the $\varepsilon \theta$-neighborhood of $S_{i}\left(\partial P_{w}(\mathcal{T}, i)\right)$, that is,

$$
R_{1}(\varepsilon, i)=\left\{y \in \mathbb{R}^{d} \mid d\left(S_{i} \circ T(y), S_{i}\left(\partial P_{w}(\mathcal{T}, i)\right)\right)<\varepsilon \theta\right\} \cap E_{v}\left(\mathcal{T}^{n}, k, \varepsilon \theta ; v, \theta\right) \cap \text { Shell }_{\boldsymbol{v}} .
$$

Remark that

$$
E_{\boldsymbol{w}}(\mathcal{T}, i, \varepsilon \theta)=\left\{z \in \mathbb{R}^{d} \mid d\left(S_{i}(z), S_{i}\left(\partial P_{\boldsymbol{w}}(\mathcal{T}, i)\right)\right)<\varepsilon \theta\right\}
$$

and it is $L_{\boldsymbol{w}}$-cylindrical. Thus we can write $R_{1}(\varepsilon, i)$ as

$$
\begin{aligned}
R_{1}(\varepsilon, i) & =T^{-1}\left(E_{\boldsymbol{w}}(\mathcal{T}, i, \varepsilon \theta)\right) \cap E_{\boldsymbol{v}}\left(\mathcal{T}^{n+1}, i, \varepsilon \theta ; v, \theta\right) \cap \text { Shell }_{\boldsymbol{v}} \\
& =\left(\pi_{\boldsymbol{w}} \circ T\right)^{-1}\left(E_{\boldsymbol{w}}(\mathcal{T}, i, \varepsilon \theta) \cap L_{\boldsymbol{w}}^{\perp}\right) \cap E_{\boldsymbol{v}}\left(\mathcal{T}^{n+1}, i, \varepsilon \theta ; v, \theta\right) \cap \text { Shell }_{\boldsymbol{v}}
\end{aligned}
$$

Let us denote the complement of $R_{1}(\varepsilon, i)$ in $E_{v}\left(\mathcal{T}^{n+1}, i, \varepsilon \theta ; v, \theta\right) \cap$ Shell $_{v}$ by

$$
R_{2}(\varepsilon, i)=E_{\boldsymbol{v}}\left(\mathcal{T}^{n+1}, i, \varepsilon \theta ; v, \theta\right) \cap \operatorname{Shell}_{\boldsymbol{v}} \backslash R_{1}(\varepsilon, i) .
$$


Lemma 16 We have

$$
\sum_{i=1}^{q} \boldsymbol{H}^{d-m+1}\left(R_{2}(\varepsilon, i)\right) \leq \boldsymbol{H}^{d-m+1}\left(E_{\boldsymbol{v}}\left(\mathcal{T}^{n}, k, s \varepsilon \theta ; v, \theta\right) \cap B_{\boldsymbol{v}}(0,1)\right) .
$$

Proof. For each point $y \in T\left(R_{2}(\varepsilon, i)\right)$, we can find a point $w \in T((\partial Q) \cap Q(i)) \subset P_{\boldsymbol{w}}(\mathcal{T}, i)$ such that $d\left(S_{i}(y), S_{i}(w)\right)<\varepsilon \theta$. Then

$$
d(y, w)<s \varepsilon \theta .
$$

Notice that the point $S_{i}(w)$ is contained in $S_{i}\left(P_{w}(\mathcal{T}, i)\right)$. On the other hand, from the definition of $R_{2}(\varepsilon, i)$, the point $S_{i}(y)$ is not contained in the set $S_{i}\left(E_{w}(\mathcal{T}, i, \varepsilon \theta)\right)$, which is nothing but the $\varepsilon \theta$-neighborhood of the boundary of $S_{i}\left(P_{w}(\mathcal{T}, i)\right)$. These imply that the point $S_{i}(y)$ is contained in $S_{i}\left(P_{w}(\mathcal{T}, i)\right)$ and that the subsets $T\left(R_{2}(\varepsilon, i)\right), 1 \leq i \leq q$, are contained in $P_{\boldsymbol{w}}(\mathcal{T}, i)$ respectively. Hence $R_{2}(\varepsilon, i), 1 \leq i \leq q$, are mutually disjoint. Since $R_{2}(\varepsilon, i)$ for $1 \leq i \leq q$ are contained in $E_{v}\left(\mathcal{T}^{n}, k, s \varepsilon \theta ; v, \theta\right) \cap B_{v}(0,1)$ from (30), we obtain (29).

In order to estimate the sum $\sum_{i=1}^{q} \boldsymbol{H}^{d-m+1}\left(R_{1}(\varepsilon, i)\right)$, we need the following lemma.

Proposition 17 There exist constants $C(d)$, which depends only on the dimension $d$, and $\gamma(\mathcal{T})>0$, which depends on $\mathcal{T}$, such that we have

$$
\sum_{k=1}^{\ell} \frac{\boldsymbol{H}^{d-m+1}\left(E_{u}(\mathcal{T}, k, \delta r) \cap B_{u}(y, r)\right)}{\boldsymbol{H}^{d-m+1}\left(B_{u}(y, r)\right)} \leq C(d) \cdot \delta \cdot M_{m+1}(\mathcal{T})
$$

for any $\boldsymbol{u} \in \mathcal{I}_{m}^{\circ}, 0<\delta \leq 4, r>0$ and any $y \in L_{\boldsymbol{u}}^{\perp} \backslash\{0\}$ satisfying $\|y\|>\gamma(\mathcal{T}) \cdot r$.

The idea behind this proposition is similar to that behind proposition 6: for each $u=$ $\left(y, u_{1}, u_{2}, \ldots, u_{m-1}\right) \in \mathcal{I}_{m}^{\circ}$, the germ of $\mathcal{D}^{m} \mathcal{T}_{u}$ at a point $z \in L_{u}^{\perp} \backslash\{0\}$ equals to the pseudodifferential $\mathcal{D}^{m+1} \mathcal{T}_{\boldsymbol{u}^{\prime}}$ of order $m+1$ at $\boldsymbol{u}^{\prime}=\left(y, u_{1}, u_{2}, \ldots, u_{m-1}, z\right)$, up to translations. The proof is almost parallel to that of proposition 6 . We assume proposition 17 for a while and finish the proof of theorem 11 . We will give a proof of proposition 17 at the end of this section.

The following estimate is a consequence of proposition 17 .

Proposition 18 There exist constants $A(d)$, which depends only on the dimension $d$, and $N(\mathcal{T})>0$, which depends on $\mathcal{T}$, such that

$$
\sum_{i=1}^{q} \frac{\boldsymbol{H}^{d-m+1}\left(R_{1}(\varepsilon, i)\right)}{\boldsymbol{H}^{d-m+1}\left(\left(E_{\boldsymbol{v}}\left(\mathcal{T}^{n}, k, 2 \theta ; v, \theta\right) \cup Q\right) \cap B_{\boldsymbol{v}}(0,1)\right)} \leq A(d) \cdot \varepsilon \cdot M_{m+1}(\mathcal{T})
$$

for $0<\varepsilon \leq 4$ and $n>N(\mathcal{T})$. 
Proof. From (27), the subset $T\left(R_{1}(\varepsilon, i)\right)$ is contained in the $s \varepsilon \theta$-neighborhood of the subset $T(Q)$. Since $T(Q)$ is $L_{w}$-cylindrical, the subsets $\pi_{w} \circ T\left(R_{1}(\varepsilon, i)\right), 1 \leq i \leq q$, are contained in

$$
X=\left\{z \in L_{\boldsymbol{w}}^{\perp} \mid d(z, T(Q)) \leq \operatorname{s\varepsilon } \theta\right\} \cap \pi_{\boldsymbol{w}} \circ T\left(\text { Shell }_{\boldsymbol{v}}\right) .
$$

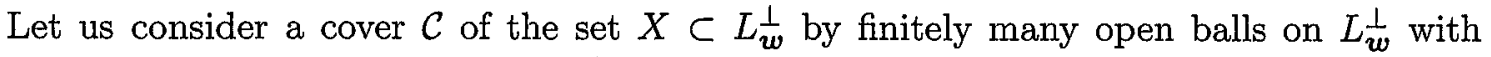
radius $\theta$. There exists a constant $\nu(d)$, which depends only on the dimension $d$, such that we can take the cover $\mathcal{C}$ so that its intersection multiplicity is not more $\nu(d)$. In fact, we can take $\nu(d)=2^{d-m+1}$ for exapmple. Also we can assume that all balls of the cover $\mathcal{C}$ intersect $X$.

Lemma 19 For any point $y$ in the balls of the cover $\mathcal{C}$, the point $y / 2$ belongs to the subset

$$
\pi_{\boldsymbol{w}} \circ T\left(\left(E_{\boldsymbol{v}}\left(\mathcal{T}^{n}, k, 2 \theta ; v, \theta\right) \cup Q\right) \cap B_{\boldsymbol{v}}(0,1)\right) .
$$

Proof. Remark that $\pi_{v} \circ T^{-1}: L_{\boldsymbol{w}}^{\perp} \rightarrow L_{v}^{\perp}$ and $\pi_{\boldsymbol{w}} \circ T: L_{\boldsymbol{v}}^{\perp} \rightarrow L_{\boldsymbol{w}}^{\perp}$ are the inverses of each other because $T\left(L_{v}\right)=L_{w}$. Also remark that $\left\|\pi_{v} \circ T^{-1}\right\| \leq\left\|T^{-1}\right\| \leq s^{n}$. Take an arbitrary point $y$ in one of balls of the cover $\mathcal{C}$. Then we can take points $z \in X$ and $w \in Q$ such that $d(y, z) \leq 2 \theta$ and that $d(z, T(w)) \leq s \varepsilon \theta$. We have, from (23),

$$
\left\|\pi_{v} \circ T^{-1}(y)\right\| \leq\left\|\pi_{v} \circ T^{-1}(z)\right\|+s^{n} \cdot 2 \theta \leq 1+s^{n} \cdot 2 \theta<2 .
$$

So $y^{\prime}=\pi_{v} \circ T^{-1}(y / 2)$ is contained in $B_{v}(0,1)$. That is, the point $y / 2$ belongs to $\pi_{w} \circ$ $T\left(B_{\boldsymbol{v}}(0,1)\right)$. On the other hand, since

$$
d(y / 2, T(w / 2)) \leq d(y / 2, z / 2)+d(z / 2, T(w) / 2) \leq(2 \theta+s \varepsilon \theta) / 2<2 \theta
$$

and $w / 2 \in Q$, the point $y / 2$ belongs to the $2 \theta$-neighborhood of $T(Q)$, which equals to $T\left(E_{v}\left(\mathcal{T}^{n}, k, 2 \theta ; v, \theta\right) \cup Q\right)$. Hence $y / 2=\pi_{\boldsymbol{w}}(y / 2)$ belongs to (33).

From the last lemma, the volumes of the union of balls in the cover $\mathcal{C}$ is not more than

$$
2^{d-m+1} \cdot \boldsymbol{H}^{d-m+1}\left(\pi_{\boldsymbol{w}} \circ T\left(E_{\boldsymbol{v}}\left(\left(\mathcal{T}^{n}, k, 2 \theta ; v, \theta\right) \cup Q\right) \cap B_{\boldsymbol{v}}(0,1)\right)\right) .
$$

Since intersection multiplicity of the cover $\mathcal{C}$ is not more than $\nu(d)$, the sum of volumes of balls in the cover $\mathcal{C}$ is not more than

$$
2^{d-m+1} \nu(d) \cdot \boldsymbol{H}^{d-m+1}\left(\pi_{\boldsymbol{w}} \circ T\left(E_{v}\left(\left(\mathcal{T}^{n}, k, 2 \theta ; v, \theta\right) \cup Q\right) \cap B_{v}(0,1)\right)\right) .
$$

Let $\gamma(\mathcal{T})$ be the constant in proposition 17. The distances from the centers of balls in $\mathcal{C}$ to the origin 0 are not smaller than $(1 / 3) \rho(\mathcal{T})^{n}-\theta$. So if $n$ is sufficiently large, they are larger than $\gamma(\mathcal{T}) \theta$ and, hence, we can apply proposition 17 to each balls in the cover with $\delta=\varepsilon \leq 4$ and $r=\theta$. As a result, we obtain

$\sum_{i=1}^{q} \frac{\boldsymbol{H}^{d-m+1}\left(E_{\boldsymbol{w}}(\mathcal{T}, i, \varepsilon \theta) \cap X\right)}{\boldsymbol{H}^{d-m+1}\left(\pi_{\boldsymbol{w}} \circ T\left(\left(E_{\boldsymbol{v}}\left(\mathcal{T}^{n}, k, 2 \theta ; v, \theta\right) \cup Q\right) \cap B_{\boldsymbol{v}}(0,1)\right)\right)} \leq 2^{d-m+1} \varepsilon \nu(d) C(d) M_{m+1}(\mathcal{T})$. 
Since $\pi_{w} \circ T\left(R_{1}(\varepsilon, i)\right), 1 \leq i \leq q$, are contained in $E_{w}(\mathcal{T}, i, \varepsilon \theta) \cap X$ respectively from (28), we obtain

$$
\sum_{i=1}^{q} \frac{\boldsymbol{H}^{d-m+1}\left(\pi_{\boldsymbol{w}} \circ T\left(R_{1}(\varepsilon, i)\right)\right)}{\boldsymbol{H}^{d-m+1}\left(\pi_{\boldsymbol{w}} \circ T\left(\left(E_{\boldsymbol{v}}\left(\mathcal{T}^{n}, k, 2 \theta ; v, \theta\right) \cup Q\right) \cap B_{\boldsymbol{v}}(0,1)\right)\right)} \leq 2^{d-m+1} \varepsilon \nu(d) C(d) M_{m+1}(\mathcal{T}) .
$$

This implies the conclusion of the proposition 18.

Now we finish the proof of proposition 14. From (29) and (32), we obtain

$$
\begin{aligned}
\sum_{i=1}^{q} \boldsymbol{H}^{d-m+1}\left(E_{\boldsymbol{v}}\left(\mathcal{T}^{n+1}, i, \varepsilon \theta ; v, \theta\right) \cap \text { Shell }_{\boldsymbol{v}}\right) \\
\leq A(d) \varepsilon \boldsymbol{M}_{m+1}(\mathcal{T}) \boldsymbol{H}^{d-m+1}\left(E_{\boldsymbol{v}}\left(\mathcal{T}^{n}, k, 2 \theta ; v, \theta\right) \cap B_{\boldsymbol{v}}(0,1)\right) \\
+A(d) \varepsilon M_{m+1}(\mathcal{T}) \boldsymbol{H}^{d-m+1}\left(Q \cap B_{\boldsymbol{v}}(0,1)\right) \\
+\boldsymbol{H}^{d-m+1}\left(E_{\boldsymbol{v}}\left(\mathcal{T}^{n}, k, s \varepsilon \theta ; v, \theta\right) \cap B_{\boldsymbol{v}}(0,1)\right)
\end{aligned}
$$

Summing up this inequality over $Q=P_{v}\left(\mathcal{T}^{n}, k, v, \theta\right), 1 \leq k \leq \ell$, and dividing both sides of the result by $\varepsilon \cdot \boldsymbol{H}^{d-m+1}\left(\operatorname{Cone}(v, \theta) \cap B_{\boldsymbol{v}}(0,1)\right)$, we obtain

$$
\begin{aligned}
\tilde{\boldsymbol{m}}_{v}\left(\mathcal{T}^{n+1}, \varepsilon ; v, \theta\right) & \leq A(d) M_{m+1}(\mathcal{T})\left(2 M_{m}\left(\mathcal{T}^{n}\right)+1\right)+s M_{m}\left(\mathcal{T}^{n}\right) \\
& \leq\left(2 A(d) M_{m+1}(\mathcal{T})+s\right) M_{m}\left(\mathcal{T}^{n}\right)+A(d) M_{m+1}(\mathcal{T})
\end{aligned}
$$

where we used the fact that the subsets $Q \cap B_{v}(0,1)$ for $Q=P_{v}\left(\mathcal{T}^{n}, k, v, \theta\right), 1 \leq k \leq \ell(n)$, have mutually disjoint interior and their union is contained in $\operatorname{Cone}(v, \theta) \cap B_{v}(0,1)$. This and lemma 15 imply proposition 14 . We completed the proof of theorem 11 and, hence, that of theorem 1 except for the proof of proposition 17 .

Here we give the proof of proposition 17 that we postponed. It is enough to prove the conclusion of the proposition for each $\boldsymbol{u}$ in $\mathcal{I}_{m}^{\circ}$, because there is only finitely many distinct maps in $\boldsymbol{D}_{m}$. So we fix $\boldsymbol{u}=\left(z, u_{1}, u_{2}, \ldots, u_{m-1}\right) \in \mathcal{I}_{m}^{\circ}$ and prove the conclusion of the proposition. Remark that each element $P_{\boldsymbol{u}}(\mathcal{T}, k)$ in the partition $\mathcal{P}_{\boldsymbol{u}}(\mathcal{T})$ is $L_{\boldsymbol{u}^{-}}$ cylindrical. Thus its intersection $P_{\boldsymbol{u}}(\mathcal{T}, k) \cap L_{\boldsymbol{u}}^{\perp}$ with $L_{\boldsymbol{u}}^{\perp}$ is $(d-m+1)$-dimensional polyhedral cone in $L_{u}^{\perp}$. We say that a ball $B_{u}(y, a)$ has regular cone property if the boundaries of $P_{u}(\mathcal{T}, k) \cap L_{u}^{\perp}, 1 \leq k \leq \ell$, are cone in that ball. (See section 2 for the definition of a cone in a ball.) We first prove the following lemma that corresponds to lemma 7 in the proof of proposition 6.

Lemma 20 There exists a constant $\gamma>0$ such that we can find a point $p(y) \in L_{u}^{\frac{1}{u}} \backslash\{0\}$ for each $y \in L_{\boldsymbol{u}}^{\perp} \backslash\{0\}$ so that $B_{\boldsymbol{u}}(p(y),\|y-p(y)\|+2 r)$ has regular cone property for $0 \leq r \leq\|y\| / \gamma$.

Proof. Let $F_{j}, 1 \leq j \leq d-m$, be the union of $j$-dimensional faces of $(d-m+1)$ dimensional polyhedral cones $P_{\boldsymbol{u}}(\mathcal{T}, k) \cap L_{\boldsymbol{u}}^{\perp}, 1 \leq k \leq \ell$. Let $\boldsymbol{S}_{\boldsymbol{u}}$ be the unit sphere on $L_{\boldsymbol{u}}^{\perp}$, 
that is, the boundary of $B_{u}(0,1)$ in $L_{u}^{\perp}$. Then $F_{1} \cap S_{u}$ consists of finitely many points. Take $\delta_{1}>0$ so small that each ball $B_{u}\left(y, 3 \delta_{1}\right)$ for $y \in F_{1} \cap \boldsymbol{S}_{\boldsymbol{u}}$ has regular cone property. Let us put $K_{1}=F_{1} \cap \boldsymbol{S}_{u}$ and $D_{1}=\cup_{z \in K_{1}} B_{\boldsymbol{u}}\left(z, \delta_{1}\right)$. By the following inductive manner, we take numbers $\delta_{j}>0$ and open sets $D_{j}$ in $L_{u}^{\frac{1}{u}}$ for $2 \leq j \leq d-m$ so that $\cup_{i=1}^{j} D_{i}$ is a neighborhood of $F_{j} \cap S_{u}$ in $L_{u}^{\perp}$. Assume that we have defined $\delta_{i}$ and $D_{i}$ for $1 \leq i \leq j-1$. Then put $K_{j}=F_{j} \cap \boldsymbol{S}_{u} \backslash \cup_{i=1}^{j-1} D_{i}$. We take $0<\delta_{j} \leq \delta_{j-1}$ so small that the ball $B\left(y, 3 \delta_{j}\right)$ for each $y \in K_{j}$ has regular cone property and put

$$
D_{j}=\cup_{y \in K_{j}} B_{u}\left(y, \delta_{j}\right) .
$$

The union $\cup_{i=1}^{j} D_{i}$ is a neighborhood of $F_{j} \cap \boldsymbol{S}_{\boldsymbol{u}}$ in $L_{\boldsymbol{u}}^{\perp}$.

We take $0<\delta<\delta_{d-m}$ so small that $2 \delta$-neighborhood of $F_{d-m} \cap \boldsymbol{S}_{\boldsymbol{u}}$ in $L_{u}^{\perp}$ is contained in $\cup_{i=1}^{d-m} D_{i}$. Then, for any point $y \in \boldsymbol{S}_{\boldsymbol{u}}$, we can find a point $q(y) \in \boldsymbol{S}_{\boldsymbol{u}}$ such that $B_{\boldsymbol{u}}(q(y),\|y-q(y)\|+2 \delta)$ has regular cone property. In fact, if $y$ is contained in the $2 \delta$-neighborhood of $F_{d-m}$, it is contained in $D_{j}$ for some $1 \leq j \leq d-m$. Hence, the choice of $q(y)$ is clear from the definition of $D_{j}$. Otherwise we can set $q(y)=y$. Then $B_{\boldsymbol{u}}(q(y),\|y-q(y)\|+2 \delta)=B(y, 2 \delta)$ does not intersect $F_{d-m}$ and has regular cone property in trivial sense.

We have proved that we can find a point $q(y)$ for each $y \in \boldsymbol{S}_{\boldsymbol{u}}$ such that $B_{\boldsymbol{u}}(q(y), \| y-$ $q(y) \|+2 \delta)$ has regular cone property. Now put $\gamma=\delta^{-1}$ and $p(y)=\|y\| q(y /\|y\|)$ for $y \in L_{u}^{\perp} \backslash\{0\}$. If $0 \leq r \leq\|y\| / \gamma$, the ball

$$
B_{u}\left(q\left(\frac{y}{\|y\|}\right),\left\|\frac{y}{\|y\|}-q\left(\frac{y}{\|y\|}\right)\right\|+\frac{2 r}{\|y\|}\right)
$$

has regular cone property because $r /\|y\| \leq \delta$. But, since each face of $P_{\boldsymbol{u}}(\mathcal{T}, k) \cap L_{\boldsymbol{u}}^{\frac{1}{u}}$ is a cone in $L_{u}^{\perp}$, this implies that $B_{\boldsymbol{u}}(p(y),\|y-p(y)\|+2 r)$ has regular cone property.

Let $\gamma$ be that in the last lemma. Let $r>0$ and consider a point $y \in L_{\boldsymbol{u}}^{\perp} \backslash\{0\}$ satisfying $\|y\|>\gamma r$. Then, from the last lemma, we can choose a point $p(y) \in L_{u}^{\perp} \backslash\{0\}$ so that $B_{u}(p(y),\|y-p(y)\|+2 r)$ has regular cone property. Let us remember the relation between the germ of the map $\mathcal{D}^{m} \mathcal{T}_{\boldsymbol{u}}$ at $p(y) \in L_{\boldsymbol{u}}^{\perp} \backslash\{0\}$ and the pseudo-differential $\mathcal{D}^{m+1} \mathcal{T}_{\boldsymbol{u}^{\prime}}$ at $\boldsymbol{u}^{\prime}=\left(z, u_{1}, u_{2}, \ldots, u_{m-1}, p(y)\right) \in \mathcal{I}_{m+1}^{\circ}$. Let $T_{u, k}$ be the linear map that coincides with $\mathcal{D}^{m} \mathcal{T}_{\boldsymbol{u}}$ on $P_{\boldsymbol{u}}(\mathcal{T}, k)$. Then we have

$$
P_{u^{\prime}}(\mathcal{T}, k)=\left\{v \in \mathbb{R}^{d} \mid p(y)+t v \in P_{u}(\mathcal{T}, k) \text { for sufficiently small } t>0 .\right\}
$$

for $1 \leq k \leq \ell$, and $\mathcal{D}^{m+1} \mathcal{T}_{u^{\prime}}=T_{u, k}$ on the interior of $P_{u^{\prime}}(\mathcal{T}, k)$. Let $\xi: \mathbb{R}^{d} \rightarrow \mathbb{R}^{d}$ be a map defined by $\xi(w)=w-p(y)$. Then we have, for $1 \leq k \leq \ell$,

$$
\left.\xi\left(P_{\boldsymbol{u}}(\mathcal{T}, k) \cap B_{\boldsymbol{u}}(p(y),\|y-p(y)\|+2 r)\right)=P_{\boldsymbol{u}^{\prime}}(\mathcal{T}, k) \cap B_{\boldsymbol{u}}(0,\|y-p(y)\|+2 r)\right) .
$$

Moreover, on each $P_{u}(\mathcal{T}, k) \cap B_{u}(p(y),\|y-p(y)\|+2 r)$, we have

$$
\mathcal{D}^{m+1} \mathcal{T}_{u^{\prime}} \circ \xi(w)=\mathcal{D}^{m} \mathcal{T}_{u}(w)+c_{k}
$$


Moreover, on each $P_{u}(\mathcal{T}, k) \cap B_{u}(p(y),\|y-p(y)\|+2 r)$, we have

$$
\mathcal{D}^{m+1} \mathcal{T}_{\boldsymbol{u}^{\prime}} \circ \xi(w)=\mathcal{D}^{m} \mathcal{T}_{\boldsymbol{u}}(w)+c_{k}
$$

for some constant $c_{k} \in \mathbb{R}^{d}$. Therefore, for $0<\delta \leq 4$, we have

$$
\xi\left(E_{u}(\mathcal{T}, k, \delta r) \cap B_{u}(y, r)\right)=E_{u^{\prime}}(\mathcal{T}, k, \delta r) \cap B_{u}(\xi(y), r)
$$

from regular cone property of the ball $B_{\boldsymbol{u}}(p(y),\|y-p(y)\|+2 r)$. Thus

$$
\boldsymbol{H}^{d-m+1}\left(E_{\boldsymbol{u}}(\mathcal{T}, k, \delta r) \cap B_{u}(y, r)\right)=\boldsymbol{H}^{d-m+1}\left(E_{\boldsymbol{u}^{\prime}}(\mathcal{T}, k, \delta r) \cap B_{u}(\xi(y), r)\right) .
$$

Let $\pi: L_{\boldsymbol{u}}^{\perp} \rightarrow L_{\boldsymbol{u}^{\prime}}^{\perp}$ be the orthogonal projection. Since $E_{\boldsymbol{u}^{\prime}}(\mathcal{T}, k, \delta r)$ is $L_{\boldsymbol{u}^{\prime}}$-cylindrical, we have

$$
\boldsymbol{H}^{d-m+1}\left(E_{\boldsymbol{u}^{\prime}}(\mathcal{T}, k, \delta r) \cap B_{\boldsymbol{u}}(\xi(y), r)\right) \leq 2 r \cdot \boldsymbol{H}^{d-m}\left(E_{\boldsymbol{u}^{\prime}}(\mathcal{T}, k, \delta r) \cap B_{\boldsymbol{u}^{\prime}}(\pi(\xi(y)), r)\right) .
$$

By repeating the part of the proof of proposition 6 after lemma 8, substituting

$$
\begin{aligned}
& d \Rightarrow d-m, \quad x \Rightarrow \pi(\xi(y)), \quad p(x) \Rightarrow 0, \quad \eta^{\prime} \Rightarrow r, \quad \varepsilon \Rightarrow \delta r \\
& E(k, \varepsilon) \Rightarrow E_{\boldsymbol{u}^{\prime}}(\mathcal{T}, k, \delta r) \quad \text { and } \\
& G\left(\eta^{\prime}, \varepsilon, x\right) \Rightarrow \sum_{k=1}^{\ell} \frac{\left.\boldsymbol{H}^{d-m}\left(E_{\boldsymbol{u}^{\prime}}(\mathcal{T}, k, \delta r) \cap B_{\boldsymbol{u}^{\prime}}(\pi(\xi(y)), r)\right)\right)}{\boldsymbol{H}^{d-m}\left(B_{\boldsymbol{u}^{\prime}}(\pi(\xi(y)), r)\right)}
\end{aligned}
$$

we can prove that there exists a constant $C^{\prime}(d)$, which depends only on the dimension $d$, such that

$$
\sum_{k=1}^{\ell} \frac{\boldsymbol{H}^{d-m}\left(E_{\boldsymbol{u}^{\prime}}(\mathcal{T}, k, \delta r) \cap B_{\boldsymbol{u}^{\prime}}(\pi(\xi(y)), r)\right)}{\boldsymbol{H}^{d-m}\left(B_{\boldsymbol{u}^{\prime}}(\pi(\xi(y)), r)\right)} \leq C^{\prime}(d) \cdot \delta \cdot M\left(\mathcal{T}, \boldsymbol{u}^{\prime}\right)
$$

Therefore we obtain

$$
\sum_{k=1}^{\ell} \frac{\boldsymbol{H}^{d-m+1}\left(E_{\boldsymbol{u}}(\mathcal{T}, k, \delta r) \cap B_{\boldsymbol{u}}(y, r)\right)}{\boldsymbol{H}^{d-m+1}\left(B_{\boldsymbol{u}}(y, r)\right)} \leq \frac{C^{\prime}(d) \cdot 2 r \boldsymbol{H}^{d-m}\left(B_{\boldsymbol{u}^{\prime}}(\pi(\xi(y)), r)\right)}{\boldsymbol{H}^{d-m}\left(B_{\boldsymbol{u}}(y, r)\right)} \delta \boldsymbol{M}\left(\mathcal{T}, \boldsymbol{u}^{\prime}\right)
$$

for $r>0,0<\delta \leq 4$ and $y \in L_{u}^{\perp} \backslash\{0\}$ satisfying $\|y\|>\gamma r$. But this implies the proposition because the fraction on the right hand side is bounded by a constant that depends only on the dimension $d$. 


\section{$9 \quad$ Proof of theorem 2}

We prove theorem 2. It is enough to prove the theorem for one of the iterations of $\mathcal{T}$. So we assume that $\mathcal{T}$ satisfies the assumption of theorem 4 by replacing $\mathcal{T}$ by its iteration if necessary. Then let $\eta>0$ be a small constant for which the conclusion of proposition 6 holds and let $\mathcal{B V}(U, \eta)$ be the space of functions introduced in section 4 . We can apply an ergodic theorem of Ionescu-Tulcea and Marinescu[6] to the Perron-Frobenius operator $P$ from proposition 9 and lemma 10 . As a result, we get the following description of the spectrum of the restriction os the Perron-Frobenius operator to $\mathcal{B V}(U, \eta),\left.P\right|_{\mathcal{B V}(U, \eta)}$ : $\mathcal{B V}(U, \eta) \rightarrow \mathcal{B V}(U, \eta)$ : the intersection of the spectrum and the unit circle $\{z \in \mathbb{C} \mid\|z\|=$ $1\}$ consists of finitely many point spectra of finite multiplicity and the other spectra are contained in a ball $\{z \in \mathbb{C} \mid\|z\|<\beta\}$ with radius $\beta<1$. Moreover, as is noted in the footnote of $[6, \mathrm{p} .143]$, we have

Lemma 21 For any $h \in \mathcal{L}^{1}(U)$, the sequence $n^{-1} \sum_{i=0}^{n-1} P^{i} h$ converges to an element of $\mathcal{B V}(U, \eta)$ in $\mathcal{L}^{1}(U)$. Especially $h \in \mathcal{B} \mathcal{V}(U, \eta)$ if $P h=h$ for $h \in \mathcal{L}^{1}(U)$.

From this lemma, the density functions of each absolutely continuous invariant probability measure is contained in the eigenspace $Z$ of $\left.P\right|_{\mathcal{B V}(U, \eta)}$ corresponding to the eigenvalue 1 . Especially there are only finitely many absolutely continuous ergodic probability measures $\mu_{i}, 1 \leq i \leq p$, for $\mathcal{T}$. Let us consider an absolutely continuous invariant probability measure $\mu$ for $\mathcal{T}$. If we decompose $\mu$ into finitely many mutually singular invariant measures, the density functions of the components are linearly independent elements in $Z$. Hence number of the components is not more than $\operatorname{dim} Z$. From this and ergodic decomposition theorem, we see that the measure $\mu$ is decomposed into finitely many ergodic components which are absolutely continuous. Therefore each absolutely continuous invariant probability measure for $\mathcal{T}$ is a convex combination of the absolutely continuous ergodic probability measures $\mu_{i}$. We proved claim (1) of theorem 2. Let $\Gamma$ be the constant in lemma 10 and let us take a number $0<\varepsilon<\min \{\eta, \eta(1-\Gamma) / \Gamma\}$.

Lemma 22 For each absolutely continuous ergodic measure $\mu_{i}$, we can find an open ball $B\left(x_{i}, \varepsilon\right)$ such that the density function $h_{i}$ of $\mu_{i}$ w.r.t. Lebesgue measure is positive $\boldsymbol{H}^{d}$-a.e. on it.

Proof. Since $P h_{i}=h_{i}$, we have $\operatorname{Osc}\left(h_{i}, \eta\right) \leq \Gamma /((1-\Gamma) \eta)$ from lemma 21 and lemma 10 . This implies

$$
\varepsilon^{-1} \int_{U} \operatorname{osc}\left(h_{i}, B(x, \varepsilon)\right) d \boldsymbol{H}^{d}(x) \leq \operatorname{Osc}\left(h_{i}, \eta\right) \leq \Gamma /((1-\Gamma) \eta)\left\|h_{i}\right\|_{L_{1}}
$$

or

$$
\int_{U} \operatorname{osc}\left(h_{i}, B(x, \varepsilon)\right) d \boldsymbol{H}^{d}(x)<\int_{U} h_{i}(x) d \boldsymbol{H}^{d}(x) .
$$


We can find a point $x \in U$ such that $\operatorname{osc}\left(h_{i}, B(x, \varepsilon)\right)<h_{i}(x)$. Then we have $h_{i}(y) \geq$ $h_{i}(x)-\operatorname{osc}\left(h_{i}, B(x, \varepsilon)\right)>0$ for $\boldsymbol{H}^{d}$-a.e. $y \in B(x, \varepsilon)$.

Let us consider open sets $W_{i}=\cup_{j=0}^{\infty} \mathcal{T}^{-j}\left(B\left(x_{i}, \varepsilon\right)\right)$ for $1 \leq i \leq p$. Then it is easy to see that $W_{i}=\operatorname{Basin}\left(\mu_{i}\right)$ modulo sets with Lebsgue measure 0 . If the closed set $Y=U \backslash \cup_{i=1}^{p} W_{i}$ had positive Lebesgue measure, the sequence $n^{-1} \sum_{i=1}^{n} P^{n}\left(\left(\boldsymbol{H}^{d}(Y)\right)^{-1} \chi_{Y}\right)$ should have converged to a linear combination of the density functions $h_{i}$ of $\mu_{i}$. But this is impossible from the invariance of $Y: T(Y) \subset Y$. So $\cup_{i=1}^{p} W_{i}$ has full measure in $U$. This implies claim(2) of theorem 2.

\section{References}

[1] Blank, M., Discreteness and continuity in problems of chaotic dynamics, Translations of Mathematical Monographs, 161. AMS, (1997).

[2] Buzzi, J.,A.C.I.M.'S for arbitrary expanding piecewise $\mathbb{R}$-analytic mappings of the plane, (preprint, IML)(1998)

[3] Buzzi, J.,A.C.I.M.'S as equilibrium states for piecewise invertible maps, (preprint, IML) (1998)

[4] Góra, P., Boyarski, A., Absolutely continuous invariant measures for piecewise expanding transformations in $\mathbb{R}^{N}$, Israel J. Math. Vol 67, 272-276, (1989)

[5] Hofbauer, F., and Keller, G., Ergodic properties of invariant measures for piecewise monotonic transformations., Math. Z. 180, 119-140 (1982)

[6] Ionescu-Tulcea,C., and Marinescu,G., Theorie ergodique pour des classes d'operations non completement continues. Ann. Math.(2) 52, 140-147 (1950)

[7] Keller, G., Propriété ergodique des endomorphismes dilatants, $C^{2}$ par morceaux, des rǵions bornées du plan, thesis, Universite de Rennes, (1979)

[8] Keller, G., Generalized bounded variation and applications to piecewise monotonic transformations, Z. Wahr. verw. Geb. 69, 461-478 (1985)

[9] Lasota, A.\& Yorke, J., On the existence of invariant measures for piecewise monotonic transformations, Trans. A.M.S., Vol 186, 481-488,(1973)

[10] Saussol, B.,Absolutely continuous invariant measures for multidimaisional expanding maps, (preprint)(1997)

[11] Tsujii, M.,Absolutely continuous invariant measures for piecewise real-analytic expanding maps on the plane., (preprint, Hokkaido University) (1998)

[12] Tsujii, M.,Piecewise expanding maps on the plane with singular ergodic properties, (preprint, Hokkaido University) (1998) To appear in Ergod. Th. E Dynam. Sys. 